\title{
Orderings of monomial ideals
}

\author{
by \\ Matthias Aschenbrenner (Berkeley, CA) and \\ Wai Yan Pong (Carson, CA)
}

\begin{abstract}
We study the set of monomial ideals in a polynomial ring as an ordered set, with the ordering given by reverse inclusion. We give a short proof of the fact that every antichain of monomial ideals is finite. Then we investigate ordinal invariants for the complexity of this ordered set. In particular, we give an interpretation of the height function in terms of the Hilbert-Samuel polynomial, and we compute bounds on the maximal order type.
\end{abstract}

\section{INTRODUCTION}

Monomial ideals (that is, ideals generated by monomials) in polynomial or power series rings play an important role in commutative algebra and algebraic combinatorics, both from a theoretical and a practical perspective. The reason for this is that more often than not problems about arbitrary ideals can be reduced to the special case of monomial ideals, and hence to questions of a combinatorial nature. Conversely, monomial ideals may be used to make algebra out of combinatorics (see, e.g., [42]). The link between monomial ideals and arbitrary ideals is provided by the theory of Gröbner bases (or standard bases); see, e.g., [6].

Let $K$ be a field and $R=K[X]=K\left[X_{1}, \ldots, X_{m}\right]$ the ring of polynomials in indeterminates $X=\left\{X_{1}, \ldots, X_{m}\right\}$ with coefficients from $K$. We employ the usual multi-index notation $X^{\nu}=X^{\nu_{1}} \cdots X^{\nu_{m}}$ for monomials, where $\nu=$ $\left(\nu_{1}, \ldots, \nu_{m}\right)$ is an $m$-tuple of non-negative integers. Divisibility of monomials in $R$ has the following well-known finiteness property:

Every sequence $X^{\nu^{(1)}}, X^{\nu^{(2)}}, \ldots, X^{\nu^{(n)}}, \ldots$ of monomials in $R$ such that $X^{\nu^{(i)}}$ does not divide $X^{\nu^{(j)}}$, for all $i<j$, is finite.

This equally elementary and fundamental fact, commonly known as "Dickson's Lemma", is arguably "the most frequently rediscovered mathematical

2000 Mathematics Subject Classification: Primary 06A06; Secondary 13D40, 13P10. 
theorem" ([6, p. 184]). Among other things, it implies Hilbert's Basis Theorem, with its numerous consequences. Recently Diane Maclagan [28] proved the following more general result:

Every sequence $I^{(1)}, I^{(2)}, \ldots, I^{(n)}, \ldots$ of monomial ideals in $R$ such that $I^{(i)} \nsupseteq I^{(j)}$ whenever $i<j$ is finite.

She also showed how this can be used to give short proofs of several other finiteness statements like the existence of a universal Gröbner basis of an ideal in $R$ and the finiteness of the number of atomic fibers of a matrix with non-negative integer entries. Galligo's theorem on the existence of generic initial ideals can also been seen as a consequence of this principle, as can the upper semi-continuity of fiber dimension (see [4]) and Sit's theorem [41] on the well-orderedness of the set of Hilbert polynomials under eventual dominance; for the latter see Section 3 of the present paper. It is these remarkable applications which seem to warrant a further investigation into combinatorial finiteness phenomena of monomials in $R$. The theory of Noetherian ordered sets provides a convenient axiomatic framework for this: Let $(S, \leq)$ be an ordered set, i.e., $S$ is a set and $\leq$ is a (partial) ordering on $S$. We call $(S, \leq)$ Noetherian if every sequence $s_{1}, s_{2}, \ldots, s_{n}, \ldots$ in $S$ such that $s_{i} \not \leq s_{j}$ for all $i<j$ is finite. Dickson's Lemma may then be rephrased as saying that the set of monomials under divisibility is Noetherian, and Maclagan's principle just expresses that the set $\mathcal{M}_{m}$ of monomial ideals in $K[X]$ ordered by reverse inclusion is Noetherian. Noetherian orderings are usually called "well-partial-orderings" or "well-quasi-orderings" in the literature (see, e.g., [25]). We follow a proposal by Joris van der Hoeven [45] and use the more concise (and perhaps more suggestive) term "Noetherian". Noetherian ordered sets play an important role in such diverse fields as asymptotic differential algebra [45], Ramsey theory [24], theoretical computer science [12], and proof theory [18].

The purpose of this paper is to study some aspects of the set of monomial ideals of $K[X]$ from the point of view of combinatorial set theory. In Section 1, after reviewing some basic facts about Noetherian ordered sets, we first give a quick proof of Maclagan's result. We also indicate a certain generalization, dealing with direct products of Noetherian ordered sets (Proposition 1.12), which was stated without proof in [28] and attributed there to Farley and Schmidt.

The complexity of a Noetherian ordered set $(S, \leq)$ can be measured in terms of certain ordinal-valued invariants. We recall their definitions and basic properties in Section 2. Here is one example: There always exists a chain in $S$ having maximal possible order type, called the height of $S$; for $x \in S$, the height of the Noetherian ordered set $S^{\nsucceq x}:=\{s \in S: s$ $\geq x\}$ is called the height of $x$ (in $S$ ). From a result of Bonnet and Pouzet 
[7] we deduce that the height of $\left(\mathcal{M}_{m}, \supseteq\right)$ is $\omega^{m}+1$. In Section 3 we give an interpretation of the height of a monomial ideal $I$ in terms of the Hilbert-Samuel polynomial of $M=R / I$. Recall that for every finitely generated graded $R$-module $M=\bigoplus_{s \in \mathbb{N}} M_{s}$, the function which associates with $s \in \mathbb{N}$ the dimension of the $K$-vector space $M_{s}$ agrees, for all sufficiently large $s$, with a polynomial in $\mathbb{Q}[T]$, called the Hilbert polynomial of $M$ (see [10, Chapter 4]). It follows that the function $s \mapsto \operatorname{dim}_{K} M_{\leq s}$, where $M_{\leq s}:=\bigoplus_{i=0}^{s} M_{i}$, also ultimately agrees with a polynomial in $\mathbb{Q}[\bar{T}]$, which is called the Hilbert-Samuel polynomial of $M$. We let $\mathcal{I}_{m}$ denote the set of homogeneous ideals of $R$, considered as a (partially) ordered set, with the ordering given by reverse inclusion. Given $I \in \mathcal{I}_{m}$ we denote the Hilbert-Samuel polynomial of $R / I$ by $p_{I}$. We totally order $\mathcal{S}_{m}=\left\{p_{I}\right.$ : $\left.I \in \mathcal{I}_{m}\right\}$ by eventual dominance: $p_{I} \leq p_{J}$ if and only if $p_{I}(s) \leq p_{J}(s)$ for all sufficiently large $s$. The map $p: \mathcal{I}_{m} \rightarrow \mathcal{S}_{m}$ that maps $I$ to $p_{I}$ is strictly increasing. It is well-known that the map taking each finitely generated $R$-module to its Hilbert polynomial is the universal additive function on finitely generated $R$-modules which is zero on modules of finite length. (See [15, Section 19.5] for a precise statement.) The following theorem, proved in Section 3 below, is in a similar spirit; it shows that $p$ is universal among strictly increasing surjections defined on the ordered set $\mathcal{I}_{m}$.

TheOREM. For every strictly increasing surjection $\varphi: \mathcal{I}_{m} \rightarrow S$, where $S$ is any totally ordered set, there exists a strictly increasing map $\psi: \mathcal{S}_{m} \rightarrow S$ with $\psi \circ p \leq \varphi$.

Every total ordering extending the ordering $\leq$ of a Noetherian ordered set $(S, \leq)$ is a well-ordering. This fact gives rise to another invariant of $(S, \leq)$ : by a theorem of de Jongh and Parikh [11], there exists a total ordering extending $\leq$ of maximal possible order type, which we call the type o $(S, \leq)$ of $(S, \leq)$. In Section 4 we obtain upper and lower bounds on o $\left(\mathcal{M}_{m}, \supseteq\right)$ : We show that

$$
\omega^{\omega^{m-1}}+1 \leq \mathrm{o}\left(\mathcal{M}_{m}, \supseteq\right) \leq \omega^{\omega^{m+1}} .
$$

The proof of the upper bound involves a generalization of a result of van den Dries and Ehrlich [43], [44] on the order type of submonoids of ordered abelian groups. The lower bound is established by studying a particularly useful total ordering on $\mathcal{M}_{m}$ extending $\supseteq$, inspired by the Kleene-Brouwer ordering of recursion theory and Kolchin's rankings of characteristic sets. Both bounds leave room for improvement.

We should mention that Dickson's Lemma and Maclagan's principle are only the first two levels of an infinite hierarchy of finiteness principles: $\mathbb{N}^{m}$ is "better-quasi-ordered." (This was proved by Nash-Williams [33].) We refer to [3] for an application of these more general finiteness properties. 
Acknowledgments. The first author would like to thank Bernd Sturmfels, whose questions on the total orderings of monomial ideals initiated this work, and Andreas Weiermann for an e-mail exchange around the topics of this paper.

Notations and conventions. The cardinality of a finite set $S$ is denoted by $|S|$. We let $m, n, \ldots$ range over $\mathbb{N}:=\{0,1,2, \ldots\}$. For any set $U$, let $U^{*}=\bigcup_{n \in \mathbb{N}} U^{n}$ denote the set of finite sequences of elements of $U$. Here $U^{0}$ consists of the single element $\varepsilon$ (the empty sequence). (So $\emptyset^{*}=\{\varepsilon\}$.) For an element $a=\left(a_{1}, \ldots, a_{n}\right) \in U^{*}$ we call the natural number $n$ the length of $a$, denoted by length $(a)$. For $a=\left(a_{1}, \ldots, a_{n}\right)$ and $b=\left(b_{1}, \ldots, b_{m}\right)$ in $U^{*}$ we write $a \sqsubseteq b$ ( $a$ is a truncation of the sequence $b$ ) if $n \leq m$ and $a=\left(a_{1}, \ldots, a_{n}\right)=\left(b_{1}, \ldots, b_{n}\right)$. By $a b:=\left(a_{1}, \ldots, a_{n}, b_{1}, \ldots, b_{m}\right)$ we denote the concatenation of the sequences $a=\left(a_{1}, \ldots, a_{n}\right)$ and $b=\left(b_{1}, \ldots, b_{m}\right)$ in $U^{*}$. If, for example, $a=\left(a_{1}\right)$, we shall also write $a_{1} b$ instead of $\left(a_{1}\right) b$. With concatenation as monoid operation, $U^{*}$ is the free monoid generated by $U$ (with identity $\varepsilon$ ). We extend concatenation to subsets of $U^{*}$ in the natural way, for example, $a S=\{a b: b \in S\}$ for $a \in U^{*}$ and $S \subseteq U^{*}$.

\section{NOETHERIAN ORDERED SETS}

In this section we first review the definitions and basic facts about Noetherian ordered sets. We then give a short proof that the set of monomial ideals in $K[X]$ is Noetherian, and outline a generalization.

Orderings and ordered sets. A quasi-ordering on a set $S$ is a binary relation $\leq$ on $S$ which is reflexive and transitive; we call $(S, \leq)$ (or simply $S$, if no confusion is possible) a quasi-ordered set. If in addition $\leq$ is antisymmetric, then $\leq$ is called an ordering, and the pair $(S, \leq)$ is called an ordered set. If $\leq$ is a quasi-ordering on $S$, then so is the inverse relation $\geq$; likewise for orderings. If $x, y$ are elements of a quasi-ordered set $S$, we write as usual $x<y$ if $x \leq y$ and $y \not \leq x$. Given an equivalence relation $\sim$ on a set $S$ which is compatible with the quasi-ordering $\leq$ on $S$, in the sense that $x \leq y \Rightarrow x^{\prime} \leq y^{\prime}$ for all $x^{\prime} \sim x$ and $y^{\prime} \sim y$, there is a unique ordering $\leq_{S / \sim}$ on the set $S / \sim=\{x / \sim: x \in S\}$ of equivalence classes of $\sim$ with

$$
x / \sim \leq_{S / \sim} y / \sim \Leftrightarrow x \leq y .
$$

If $\leq$ is an ordering on $S$, then $\leq_{S / \sim}$ is an ordering on $S / \sim$. For any quasiordering $\leq$ on $S$, the equivalence relation on $S$ defined by

$$
x \sim y \Leftrightarrow x \leq y \text { and } y \leq x
$$

is compatible with $\leq$, and in this case $\leq_{S / \sim}$ is an ordering. Hence by passing from $(S, \leq)$ to $\left(S / \sim, \leq_{S / \sim}\right)$ if necessary, we can usually reduce the study of 
quasi-orderings to the one of orderings. In the following, we shall therefore concentrate on ordered sets.

Total orderings and directed orderings. We say that an ordering on a set $S$ is total if $x \leq y$ or $y \leq x$ for all $x, y \in S$. An ordering $\leq^{\prime}$ on a set $S$ is said to extend the ordering $\leq$ on $S$ if $x \leq y \Rightarrow x \leq^{\prime} y$ for all $x, y \in S$. Every ordering on a set $S$ can be extended to a total ordering on $S$ (Szpilrajn's Theorem; the proof uses the Ultrafilter Axiom). An ordering on $S$ is directed if for any $x, y \in S$ there exists $z \in S$ with $x \leq z$ and $y \leq z$. Any total ordering is directed.

Maps between ordered sets. A map $\varphi: S \rightarrow T$ between ordered sets $S$ and $T$ is called increasing if

$$
x \leq y \Rightarrow \varphi(x) \leq \varphi(y) \text { for all } x, y \in S
$$

and decreasing if

$$
x \leq y \Rightarrow \varphi(x) \geq \varphi(y) \quad \text { for all } x, y \in S .
$$

Similarly, we say that $\varphi: S \rightarrow T$ is strictly increasing if

$$
x<y \Rightarrow \varphi(x)<\varphi(y) \text { for all } x, y \in S
$$

and strictly decreasing if

$$
x<y \Rightarrow \varphi(x)>\varphi(y) \quad \text { for all } x, y \in S .
$$

We shall write $\operatorname{Incr}(S, T)$ for the set of all increasing maps $S \rightarrow T$ and $\operatorname{Decr}(S, T)$ for the set of all decreasing maps $S \rightarrow T$. A map $\psi: S \rightarrow T$ is a quasi-embedding of $S$ into $T$ if

$$
\psi(x) \leq \psi(y) \Rightarrow x \leq y \quad \text { for all } x, y \in S .
$$

An increasing quasi-embedding $S \rightarrow T$ is called an embedding of $S$ into $T$. Finally, a map $S \rightarrow T$ is called an isomorphism between $S$ and $T$ if it is increasing and bijective, and its inverse is also increasing.

Construction of ordered sets. Every set $S$ can be equipped with the trivial ordering, given by $x \leq y \Leftrightarrow x=y$. There are a number of standard constructions for obtaining new (quasi-) ordered sets from given ones. For example, by restricting the ordering, any subset of an ordered set can be construed as an ordered set in its own right. Let us explicitly mention some of the constructions used below. For this, let $\left(S, \leq_{S}\right)$ and $\left(T, \leq_{T}\right)$ be ordered sets. The disjoint union of the sets $S$ and $T$ is naturally ordered by the relation $\leq_{S} \cup \leq_{T}$; we shall denote this ordered set by $S \amalg T$. The cartesian product $S \times T$ of $S$ and $T$ can be made into an ordered set by means of the product ordering:

$$
(x, y) \leq\left(x^{\prime}, y^{\prime}\right): \Leftrightarrow x \leq_{S} x^{\prime} \text { and } y \leq_{T} y^{\prime},
$$


or the lexicographic ordering:

$$
(x, y) \leq_{\text {lex }}\left(x^{\prime}, y^{\prime}\right): \Leftrightarrow x<_{S} x^{\prime} \text { or }\left(x=x^{\prime} \text { and } y \leq_{T} y^{\prime}\right),
$$

for $(x, y),\left(x^{\prime}, y^{\prime}\right) \in S \times T$. Taking $S=T$ yields the product ordering and the lexicographic ordering on $T^{2}=T \times T$, and by repeating the construction, on $T^{m}$ for any $m>0$. More generally, if $I$ is any set, then the set $T^{I}$ of all functions $I \rightarrow T$ is ordered by setting

$$
f \leq g: \Leftrightarrow f(i) \leq_{T} g(i) \text { for all } i \in I .
$$

By restriction this yields orderings on the subsets $\operatorname{Incr}(S, T)$ and $\operatorname{Decr}(S, T)$ of $T^{S}$. If the ordering on $S$ is directed, we have (at least) two other ways of defining a quasi-ordering on $T^{S}$ which extends the product ordering:

(1) using the lexicographic ordering, defined by

$$
\begin{aligned}
f \leq_{\operatorname{lex}} g: \Leftrightarrow & f=g, \text { or there is } y \in S \text { with } \\
& f(x)=g(x) \text { for all } x<_{S} y \text { and } f(y)<_{T} g(y),
\end{aligned}
$$

and

(2) using the dominance quasi-ordering, given by

$$
f \preceq g: \Leftrightarrow \text { there is } y \in S \text { with } f(x) \leq_{T} g(x) \text { for all } x \geq_{S} y .
$$

If both $\leq_{S}$ and $\leq_{T}$ are total, then $\leq_{\text {lex }}$ is total. In general, the dominance quasi-ordering is neither antisymmetric (i.e., not an ordering on $T^{S}$ ) nor total.

EXAMPLE 1.1. We consider $\mathbb{N}$ as an ordered set under its usual ordering, and we equip $\mathbb{N}^{m}$ with the product ordering. For $\nu=\left(\nu_{1}, \ldots, \nu_{m}\right) \in \mathbb{N}^{m}$ we put $|\nu|=\nu_{1}+\cdots+\nu_{m}$ (the degree of $\nu$ ). Let $X=\left\{X_{1}, \ldots, X_{m}\right\}$ be distinct indeterminates and $X^{\diamond}=\left\{X^{\nu}: \nu \in \mathbb{N}^{m}\right\}$ the free commutative monoid generated by $X$, where $X^{\nu}:=X_{1}^{\nu_{1}} \cdots X_{m}^{\nu_{m}}$ for $\nu=\left(\nu_{1}, \ldots, \nu_{m}\right) \in \mathbb{N}^{m}$. We order $X^{\diamond}$ by divisibility:

$$
X^{\nu} \leq X^{\mu}: \Leftrightarrow \mu=\nu+\lambda \text { for some } \lambda \in \mathbb{N}^{m} .
$$

Then $\nu \mapsto X^{\nu}: \mathbb{N}^{m} \rightarrow X^{\diamond}$ is an isomorphism of ordered sets. The elements of $X^{\diamond}$ can be seen as monomials in the polynomial ring $K[X]=$ $K\left[X_{1}, \ldots, X_{m}\right]$, where $K$ is a field. Here, the identity element $\varepsilon$ of $X^{\diamond}$ is identified with the monomial 1.

Final segments and antichains. A final segment of an ordered set $(S, \leq)$ is a subset $F \subseteq S$ such that

$$
x \leq y \wedge x \in F \Rightarrow y \in F, \quad \text { for all } x, y \in S .
$$

(Dually, $I \subseteq S$ is called an initial segment if $S \backslash I$ is a final segment.) Given an arbitrary subset $X$ of $S$, we denote by

$$
(X):=\{y \in S: \exists x \in X(x \leq y)\}
$$


the final segment generated by $X$. We construe the set $\mathcal{F}(S)$ of final segments of $S$ as an ordered set, with the ordering given by reverse inclusion.

EXAMPLE 1.2. Under the isomorphism in Example 1.1, final segments of $\mathbb{N}^{m}$ correspond to ideals in the commutative monoid $X^{\diamond}$, that is, subsets $I \subseteq X^{\diamond}$ such that $v u \in I$ for all $u \in I$ and $v \in X^{\diamond}$. Considering the elements of $X^{\diamond}$ as monomials in a polynomial ring $K[X]$ over a field $K$, the ordered set $\mathcal{F}\left(\mathbb{N}^{m}\right)$ becomes isomorphic to the set of monomial ideals of $K[X]$ (that is, ideals of $K[X]$ which are generated by monomials), ordered by reverse inclusion.

We write $x \| y$ if $x, y \in S$ are incomparable, that is, if $x \not \leq y$ and $y \not \leq x$. An antichain of $S$ is a subset $A \subseteq S$ such that any two distinct elements $x$ and $y$ of $A$ are incomparable. (For example, a generating set of a final segment $F$ of $S$ is a minimal generating set for $F$ if and only if it is an antichain.) A subset $C$ of $S$ is called a chain if the restriction of the ordering of $S$ to $C$ is total, that is, if for all $x, y \in C$ we have $x \leq y$ or $y \leq x$.

Noetherian orderings. An ordered set $S$ is well-founded if there is no infinite strictly decreasing sequence $x_{0}>x_{1}>\cdots$ in $S$. We say that an ordered set $S$ is Noetherian if it is well-founded and every antichain of $S$ is finite. For example, every finite ordered set is Noetherian. Since every antichain of a totally ordered set consists of at most one element, a totally ordered set $S$ is Noetherian if and only if it is well-founded; in this case $S$ is called well-ordered. For every well-ordered set $S$ there exists a unique ordinal number, called the order type o $(S)$ of $S$, which is isomorphic to $S$.

An infinite sequence $x_{0}, x_{1}, \ldots$ in $S$ is $\operatorname{good}$ if $x_{i} \leq x_{j}$ for some $i<j$, and bad otherwise. (For instance, if $\left\{x_{0}, x_{1}, \ldots\right\}$ is an antichain, then $x_{0}, x_{1}, \ldots$ is bad.) The following characterization of Noetherian orderings is folklore; we omit the proof. (For the details, see, e.g., [3].)

Proposition 1.3. The following are equivalent, for an ordered set $S$ :

(1) $S$ is Noetherian.

(2) Every infinite sequence $x_{0}, x_{1}, \ldots$ in $S$ contains an increasing subsequence.

(3) Every infinite sequence $x_{0}, x_{1}, \ldots$ in $S$ is good.

(4) Any final segment of $S$ is finitely generated.

(5) $(\mathcal{F}(S), \supseteq)$ is well-founded (i.e., the ascending chain condition with respect to inclusion holds for final segments of $S)$.

(6) Every total ordering on $S$ which extends $\leq$ is a well-ordering.

The proposition immediately implies: 
ExAmPLES 1.4. Let $S$ and $T$ be ordered sets.

(1) If there exists an increasing surjection $S \rightarrow T$, and $S$ is Noetherian, then so is $T$. In particular: If $S$ is Noetherian, then any ordering on $S$ which extends the given ordering is Noetherian; if $S$ is Noetherian and $\sim$ is an equivalence relation on $S$ which is compatible with the ordering of $S$, then $S / \sim$ is Noetherian.

(2) If there exists a quasi-embedding $S \rightarrow T$, and $T$ is Noetherian, then $S$ is Noetherian. In particular, if $T$ is Noetherian, then any subset of $T$ with the induced ordering is Noetherian.

(3) If $S$ and $T$ are Noetherian and $U$ is an ordered set which contains both ordered sets $S$ and $T$, then $S \cup T$ is Noetherian. In particular, it follows that $S \amalg T$ is Noetherian.

(4) If $S$ and $T$ are Noetherian, then so is $S \times T$ with the product ordering. Inductively, it follows that if the ordered set $S$ is Noetherian, then so is $S^{m}$ equipped with the product ordering, for every $m$. In particular, for each $m$, the ordered set $\mathbb{N}^{m}$ is Noetherian ("Dickson's Lemma").

For future use we also remark:

LEMmA 1.5. Let $\varphi: S \rightarrow T$ be a map between ordered sets $S$ and $T$, with $S$ Noetherian.

(1) If $\varphi$ is strictly increasing, then $\varphi$ has finite fibers.

(2) If $\varphi$ is decreasing, and $T$ is well-founded, then the image of $\varphi$ is finite.

Noetherianity of the set of monomial ideals. By Proposition 1.3, if $S$ is Noetherian, then the ordered set $\mathcal{F}(S)$ of final segments of $S$ is well-founded. In general, it is not true that if $S$ is Noetherian, then $\mathcal{F}(S)$ is Noetherian. A counterexample was found by Rado [36]. (This example is indeed "generic" in the sense that a Noetherian ordered set $S$ contains an isomorphic copy of it if and only if $\mathcal{F}(S)$ is non-Noetherian; see, e.g., [3].) We will now give a short proof of the fact that the ordered set $\mathcal{F}\left(\mathbb{N}^{m}\right)$ of monomial ideals is Noetherian. Here is a key observation:

Lemma 1.6. $\mathcal{F}(S \times T) \cong \operatorname{Decr}(S, \mathcal{F}(T))$, for ordered sets $S$ and $T$.

Proof. For a final segment $F \in \mathcal{F}(S \times T)$ let $\varphi_{F}: S \rightarrow \mathcal{F}(T)$ be defined by $\varphi_{F}(x)=\{y \in T:(x, y) \in F\}$ for $x \in S$. It is straightforward to verify that $\varphi_{F}$ is decreasing, and $F \mapsto \varphi_{F}$ is an isomorphism $\mathcal{F}(S \times T) \rightarrow$ $\operatorname{Decr}(S, \mathcal{F}(T))$.

In particular, we have $\mathcal{F}\left(\mathbb{N}^{m}\right) \cong \operatorname{Decr}\left(\mathbb{N}, \mathcal{F}\left(\mathbb{N}^{m-1}\right)\right)$ for $m>0$. This fact allows us to analyze $\mathcal{F}\left(\mathbb{N}^{m}\right)$ by induction on $m$; it also makes it necessary to take a closer look at decreasing maps $\mathbb{N} \rightarrow \mathcal{F}\left(\mathbb{N}^{m-1}\right)$. More generally, for any ordered set $S$, let us use $S^{(\geq)}$to denote the set $\operatorname{Decr}(\mathbb{N}, S)$ of all 
infinite decreasing sequences $s=\left(s_{0}, s_{1}, \ldots\right)$ of elements $s_{0} \geq s_{1} \geq \cdots$ of $S$, ordered componentwise (that is, by restriction of the product ordering on $S^{\mathbb{N}}$ to $\left.S^{(\geq)}\right)$.

Proposition 1.7. If $S$ is Noetherian, then so is $S^{(\geq)}$.

Proof. The proof is inspired by Nash-Williams's proof [32] of Higman's Lemma (see Lemma 1.9 below). Assume to the contrary that $S$ is Noetherian and $s^{(0)}, s^{(1)}, \ldots$ is a bad sequence in $S^{(\geq)}$; we write $s^{(i)}=\left(s_{0}^{(i)}, s_{1}^{(i)}, \ldots\right)$. Every sequence $s=\left(s_{0}, s_{1}, \ldots\right)$ in $S^{(\geq)}$becomes eventually stationary; we let $j(s)$ denote the smallest index $j \in \mathbb{N}$ such that $s_{j}=s_{j+1}=\cdots$. We may assume that the bad sequence is chosen in such a way that for every $i, j\left(s^{(i)}\right)$ is minimal among the $j(s)$, where $s$ ranges over all elements of $S^{(\geq)}$with the property that $s^{(0)}, s^{(1)}, \ldots, s^{(i-1)}, s$ can be continued to a bad sequence in $S^{(\geq)}$. We may further assume that there is an index $i_{0}$ such that $j\left(s^{(i)}\right)>0$ for all $i \geq i_{0}$. Now consider the sequence $s_{0}^{\left(i_{0}\right)}, s_{0}^{\left(i_{0}+1\right)}, \ldots$ in $S$. Since $S$ is Noetherian, there exists an infinite sequence $i_{0} \leq i_{1}<i_{2}<\cdots$ of indices such that $s_{0}^{\left(i_{1}\right)} \leq s_{0}^{\left(i_{2}\right)} \leq \ldots$. Put $t^{\left(i_{k}\right)}:=\left(s_{1}^{\left(i_{k}\right)}, s_{2}^{\left(i_{k}\right)}, \ldots\right)$ for all $k>0$. It is now easily seen that then $s^{(0)}, \ldots, s^{\left(i_{1}-1\right)}, t^{\left(i_{1}\right)}, t^{\left(i_{2}\right)}, \ldots$ is a bad sequence in $S^{(\geq)}$. But $j\left(t^{\left(i_{k}\right)}\right)=j\left(s^{\left(i_{k}\right)}\right)-1$, contradicting the minimality property of our original bad sequence.

The ordered set $\mathcal{F}(\mathbb{N})$ is clearly well-ordered (of order type $\omega+1$ ), hence Noetherian. This is the base case for an induction on $m$, which yields, using Proposition 1.7:

COROLlary 1.8. The set of monomial ideals in $K\left[X_{1}, \ldots, X_{m}\right]$, ordered by reverse inclusion, is Noetherian, for any $m$.

See [28] for another proof of this result, using primary decomposition of monomial ideals.

Higman's lemma. In the following, we will often make use of a fact due to Higman [19]. Let $S$ be an ordered set. We define an ordering on the set $S^{*}$ of finite sequences of elements of $S$ as follows:

$$
\left(x_{1}, \ldots, x_{m}\right) \leq^{*}\left(y_{1}, \ldots, y_{n}\right): \Leftrightarrow\left\{\begin{array}{l}
\text { there exists a strictly increas- } \\
\text { ing function } \varphi:\{1, \ldots, m\} \rightarrow \\
\{1, \ldots, n\} \text { such that } x_{i} \leq y_{\varphi(i)} \\
\text { for all } 1 \leq i \leq m .
\end{array}\right.
$$

Lemma 1.9 (Higman). If $S$ is Noetherian, then the ordering $\leq^{*}$ on $S^{*}$ is Noetherian. 
The equivalence relation $\sim$ on $S^{*}$ defined by

$$
\left(x_{1}, \ldots, x_{m}\right) \sim\left(y_{1}, \ldots, y_{n}\right): \Leftrightarrow\left\{\begin{array}{l}
m=n \& \text { there exists a permu- } \\
\text { tation } \sigma \text { of }\{1, \ldots, m\} \text { such that } \\
x_{i}=y_{\sigma(i)} \text { for all } 1 \leq i \leq m
\end{array}\right.
$$

is compatible with $\leq^{*}$, and hence induces an ordering on $S^{\diamond}:=S^{*} / \sim$, which we denote by $\leq^{\diamond}$. By Higman's Lemma, if $(S, \leq)$ is Noetherian, then so is $\left(S^{\diamond}, \leq^{\diamond}\right)$. For $x=\left(x_{1}, \ldots, x_{m}\right) \in S^{*}$ we denote by $[x]=\left[x_{1}, \ldots, x_{m}\right] \in S^{\diamond}$ the equivalence class of $x$, and we put $|w|=m$ for $w=\left[x_{1}, \ldots, x_{m}\right] \in S^{\diamond}$. We may think of the elements of $S^{*}$ as non-commutative words in the alphabet $S$, and of the elements of $S^{\diamond}$ as commutative words in $S$. Note that $S^{\diamond}$, with concatenation of commutative words, is the free commutative monoid generated by $S$.

REMark 1.10. We identify $S$ with a subset of $S^{\diamond}$ in a natural way. Let us call a total ordering $\leq$ of $S^{\diamond}$ extending the ordering on $S$ a term ordering if $\varepsilon \leq v$ and $v \leq w \Rightarrow s v \leq s w$, for all $v, w \in S^{\diamond}$ and $s \in S$. Then, for $v, w \in S^{\diamond}$,

$$
v \leq^{\diamond} w \Leftrightarrow v \leq w \text { for all term orderings } \leq \text { of } S^{\diamond} .
$$

This follows, e.g., from the Artin-Schreier theory of formally real fields applied to the quotient field of the monoid ring $\mathbb{Q}\left[S^{\diamond}\right]$. In the case $S=X=$ $\left\{X_{1}, \ldots, X_{m}\right\}$ ordered such that $X_{1}<\cdots<X_{m}$, monomial ideals of $K[X]$ whose corresponding final segment $E \in \mathcal{F}\left(\mathbb{N}^{m}\right)$ is also a final segment with respect to $\leq^{\diamond}$ are called strongly stable.

REMARK 1.11. If $(S, \leq)$ is Noetherian, then so is any ordering on $S^{\diamond}$ which extends $\leq^{\diamond}$. An important example is the multiset ordering on $S^{\diamond}$, defined by

$$
s \npreceq t: \Leftrightarrow\left\{\begin{array}{l}
s=t, \text { or for each } i \in\{1, \ldots, n\} \\
\text { there exists } j \in\{1, \ldots, l\} \text { with } \\
s_{m+i} \leq t_{m+j},
\end{array}\right.
$$

where we write $s=\left[s_{1}, \ldots, s_{m+n}\right], t=\left[t_{1}, \ldots, t_{m+l}\right]$ with $s_{i}=t_{i}$ for $1 \leq i$ $\leq m$ and $\left\{s_{m+1}, \ldots, s_{m+n}\right\} \cap\left\{t_{m+1}, \ldots, t_{m+l}\right\}=\emptyset$. To show that

$$
s \leq^{\diamond} t \Rightarrow s \npreceq t
$$

we proceed by induction on $m$, the case $m=0$ being trivial. Suppose $m>0$ and $s \leq \diamond t$. Then there exists an injective function

$$
\varphi:\{1, \ldots, m+n\} \rightarrow\{1, \ldots, m+l\}
$$

such that $s_{k} \leq t_{\varphi(k)}$ for all $1 \leq k \leq m+n$. Given $i$ with $1 \leq i \leq n$, we have to show that $s_{m+i} \leq t_{m+j}$ for some $j$ with $1 \leq j \leq l$. Injectivity of $\varphi$ implies 
that there exists $r \in \mathbb{N}$ such that

$$
\varphi(m+i), \varphi^{2}(m+i)=\varphi(\varphi(m+i)), \ldots, \varphi^{r}(m+i) \in\{1, \ldots, m\}
$$

and $\varphi^{r+1}(m+i)>m$. Hence $j=m-\varphi^{r+1}(m+i)$ works.

A generalization. The ideas used to establish Noetherianity of $\mathcal{F}\left(\mathbb{N}^{m}\right)$ above can be generalized somewhat to give a proof of the following fact:

Proposition 1.12. Let $S$ and $T$ be ordered sets. If $\mathcal{F}(S)$ and $\mathcal{F}(T)$ are Noetherian, then $\mathcal{F}(S \times T)$ is Noetherian.

Recall that for ordered sets $S$ and $T$, we use $\operatorname{Decr}(S, T)$ to denote the set of all decreasing maps $S \rightarrow T$, ordered pointwise: $\varphi \leq \psi \Leftrightarrow \varphi(x) \leq \psi(x)$ for all $x \in S$.

Lemma 1.13. Let $S \neq \emptyset$ be an ordered set. The following are equivalent:

(1) For every Noetherian ordered set $T, \operatorname{Decr}(S, T)$ is Noetherian.

(2) For some Noetherian ordered set $T$ with $|T|>1, \operatorname{Decr}(S, T)$ is Noetherian.

(3) $\operatorname{Decr}(S, 2)$ is Noetherian. (Here $2=\{0,1\}$ with $0<1$.)

(4) $\mathcal{F}(S)$ is Noetherian.

Proof. $(1) \Rightarrow(2)$ is trivial. For $(2) \Rightarrow(3)$, let $T$ be a Noetherian ordered set with more than 1 element, such that $\operatorname{Decr}(S, T)$ is Noetherian. If $T$ is an antichain, then so is $\operatorname{Decr}(S, T)$. Hence $\operatorname{Decr}(S, T)$, and thus $S$, are finite. Therefore $\operatorname{Decr}(S, 2)$ is finite, hence Noetherian. If $T$ is not an antichain, then there exists a quasi-embedding $\operatorname{Decr}(S, 2) \rightarrow \operatorname{Decr}(S, T)$, showing that $\operatorname{Decr}(S, 2)$ is Noetherian. For $(3) \Rightarrow(4)$, note that for every $F \in \mathcal{F}(S)$, the characteristic function $\varphi_{F}: S \rightarrow 2=\{0,1\}$ of $S \backslash F$, given by

$$
\varphi_{F}(x)= \begin{cases}0 & \text { if } x \in F, \\ 1 & \text { if } x \notin F,\end{cases}
$$

is decreasing, and $\varphi_{F} \leq \varphi_{G}$ if and only if $F \supseteq G$, for $F, G \in \mathcal{F}(S)$. For (4) $\Rightarrow(1)$, suppose that $\mathcal{F}=\mathcal{F}(S)$ is Noetherian, and let $T$ be a Noetherian ordered set. Then the image of every decreasing map $S \rightarrow T$ is finite (Lemma 1.5(2)). For $\varphi \in \operatorname{Decr}(S, T)$ and $y \in T$, the inverse image $\varphi^{-1}\left(T_{y}\right)$ of the initial segment $T_{y}=T \backslash(y)=\{z \in T: z \nsupseteq y\}$ of $T$ is a final segment of $S$. We define a map

$$
\Psi: \operatorname{Decr}(S, T) \rightarrow(T \times \mathcal{F})^{\diamond}
$$

as follows: Given $\varphi \in \operatorname{Decr}(S, T)$ let $y_{1}, \ldots, y_{k} \in T$ be the distinct elements of $\varphi(S)$; hence $\varphi^{-1}\left(T_{y_{i}}\right) \supseteq \varphi^{-1}\left(T_{y_{j}}\right)$ if $y_{i}>y_{j}$. We put

$$
\Psi(\varphi)=\left[\left(y_{1}, \varphi^{-1}\left(T_{y_{1}}\right)\right), \ldots,\left(y_{k}, \varphi^{-1}\left(T_{y_{k}}\right)\right)\right] .
$$


One checks easily that $\Psi$ is a quasi-embedding, where $(T \times \mathcal{F})^{\diamond}$ is equipped with the ordering $\leq^{\diamond}$. Since $(T \times \mathcal{F})^{\diamond}$ is Noetherian, so is $\operatorname{Decr}(S, T)$, as desired.

REMARK 1.14. In [8, Lemma 2.12] it is shown that if $S$ is Noetherian and $T$ is well-founded, then $\operatorname{Decr}(S, T)$ is well-founded.

Proposition 1.12 now follows from Lemmas 1.6 and 1.13.

\section{INVARIANTS OF NOETHERIAN ORDERED SETS}

Here, we introduce certain ordinal numbers associated to Noetherian ordered sets, and establish (or recall) some fundamental facts about them. After some preliminaries concerning ordinal arithmetic we discuss the height of a Noetherian, or more generally well-founded, ordered set. We then define the type and width of a Noetherian ordered set $S$ in terms of the heights of certain well-founded trees associated to $S$, and we state some of the basic relations between them. We relate another invariant (the minimal order type of $S$ ) with the height, and we compute the height of a certain modification of one of the trees associated with $S$. We finish by computing these invariants for the ordered set $S=\mathbb{N}^{m}$.

Natural sum and product of ordinals. We denote the class of all ordinal numbers by On. We identify each ordinal with the set of its predecessors; thus $\alpha<\beta$ is synonymous with $\alpha \in \beta$, for $\alpha, \beta \in$ On. The smallest infinite ordinal is denoted by $\omega$. Any non-zero ordinal $\alpha$ can be expressed in the form

$$
\alpha=\omega^{\gamma_{1}} a_{1}+\omega^{\gamma_{2}} a_{2}+\cdots+\omega^{\gamma_{n}} a_{n},
$$

where $\gamma_{1}>\gamma_{2}>\cdots>\gamma_{n}$ are ordinals and $a_{1}, \ldots, a_{n} \in \mathbb{N}$. If we require in addition that the $a_{i}$ are positive, then this representation of $\alpha$ is unique and called the Cantor normal form of $\alpha$. The (Hessenberg) natural sum $\alpha \oplus \beta$ of two ordinals

$$
\alpha=\omega^{\gamma_{1}} a_{1}+\omega^{\gamma_{2}} a_{2}+\cdots+\omega^{\gamma_{n}} a_{n}
$$

and

$$
\beta=\omega^{\gamma_{1}} b_{1}+\omega^{\gamma_{2}} b_{2}+\cdots+\omega^{\gamma_{n}} b_{n}
$$

(where $a_{i}, b_{j} \in \mathbb{N}$ ) is defined by

$$
\alpha \oplus \beta=\omega^{\gamma_{1}}\left(a_{1}+b_{1}\right)+\omega^{\gamma_{2}}\left(a_{2}+b_{2}\right)+\cdots+\omega^{\gamma_{n}}\left(a_{n}+b_{n}\right) .
$$

In particular, we have $0 \oplus \alpha=\alpha \oplus 0=\alpha$ for all $\alpha \in$ On. The operation $\oplus$ on On is associative, commutative and strictly increasing when one of the arguments is fixed: $\alpha<\beta \Rightarrow \alpha \oplus \gamma<\beta \oplus \gamma$, for all $\alpha, \beta, \gamma \in$ On. It follows that $\oplus$ is cancellative (i.e., $\alpha \oplus \gamma=\beta \oplus \gamma \Rightarrow \alpha=\beta$ ). The natural product of 
ordinals $\alpha$ and $\beta$ written as in (2.1) and (2.2) above, respectively, is given by

$$
\alpha \otimes \beta=\bigoplus_{i, j} \omega^{\gamma_{i} \oplus \gamma_{j}} a_{i} b_{j}
$$

The natural product, too, is associative, commutative, and strictly increasing in both arguments (hence cancellative). The distributive law for $\oplus$ and $\otimes$ holds: $\alpha \otimes(\beta \oplus \gamma)=(\alpha \otimes \beta) \oplus(\alpha \otimes \gamma)$. We refer to [5] for more information about the natural operations on On. Below, we will make use of the identity

$$
\alpha \oplus \beta=\sup \left\{\alpha^{\prime} \oplus \beta+1, \alpha \oplus \beta^{\prime}+1: \alpha^{\prime}<\alpha, \beta^{\prime}<\beta\right\}
$$

for ordinals $\alpha, \beta$.

Height functions. Let $S$ be a well-founded ordered set. For a proof of the following lemma see, e.g., $[16, \S 2.7]$. By convention, $\sup \emptyset:=0 \in$ On.

LEMMA 2.1. The following are equivalent, for a map $h: S \rightarrow$ On:

(1) $h$ is strictly increasing, and if $h^{\prime}: S \rightarrow$ On is a strictly increasing map, then $h(x) \leq h^{\prime}(x)$ for all $x \in S$.

(2) $h$ is strictly increasing, and $h(S)$ is an initial segment of On.

(3) $h(x)=\sup \{h(y)+1: y<x\}$ for all $x \in S$.

There exists a unique function $h=\mathrm{ht}_{S}: S \rightarrow$ On satisfying the equivalent conditions of the lemma. If $S$ is clear from the context, we shall just write ht for ht $S$. The ordinal ht $(x)$ is called the height of $x$ in $S$, and the image

$$
\operatorname{ht}(S)=\sup \{\operatorname{ht}(x)+1: x \in S\} \in \text { On }
$$

of $S$ under ht is called the height of the well-founded ordered set $S$. The height of $S$ is the smallest ordinal $\alpha$ such that there exists a strictly increasing function $S \rightarrow \alpha$. Equivalently:

$$
\operatorname{ht}(S)=\sup \{\mathrm{o}(C): C \subseteq S \text { chain }\} .
$$

If $S$ is well-ordered, then the height $\operatorname{ht}(S)$ of $S$ agrees with the order type o $(S)$ of $S$, and the height function ht: $S \rightarrow \operatorname{ht}(S)$ is the unique isomorphism $S \rightarrow \mathrm{o}(S)$.

LEMMA 2.2. Let $S$ and $T$ be non-empty well-founded ordered sets.

(1) If there exists a strictly increasing map $S \rightarrow T$, then $\operatorname{ht}(S) \leq \operatorname{ht}(T)$.

(2) $\operatorname{ht}(S \amalg T)=\max (\operatorname{ht}(S), \operatorname{ht}(T))$.

(3) $\operatorname{ht}_{S \times T}(s, t)=\operatorname{ht}_{S}(s) \oplus \mathrm{ht}_{T}(t)$ for all $s \in S, t \in T$.

(4) $\max (\mathrm{ht}(S), \mathrm{ht}(T)) \leq \mathrm{ht}(S \times T)<\mathrm{ht}(S) \oplus \mathrm{ht}(T)$.

Proof. Part (1) follows immediately from (1) in the previous lemma. Part (2) is obvious. For a proof of (3) and (4) see [16, 4.8.3].

The following lemma will be used in Section 3. Let us call a map $\varphi: S \rightarrow T$ between ordered sets $S$ and $T$ non-decreasing if $x<y \Rightarrow \varphi(x) \nsupseteq \varphi(y)$, for 
all $x, y \in S$. (If $T$ is totally ordered, then non-decreasing is equivalent to strictly increasing.)

Lemma 2.3. Let $S$ be a well-founded ordered set. The following are equivalent, for a strictly increasing surjection $h: S \rightarrow T$, where $T$ is a well-ordered set:

(1) $\mathrm{ht}_{S}=\mathrm{ht}_{T} \circ \mathrm{h}$.

(2) For every ordered set $T^{\prime}$ and strictly increasing map $h^{\prime}: S \rightarrow T^{\prime}$ there exists a non-decreasing map $\psi: T \rightarrow T^{\prime}$ such that $\psi \circ h \leq h^{\prime}$.

(3) For every totally ordered set $T^{\prime}$ and strictly increasing map $h^{\prime}: S \rightarrow T^{\prime}$ there exists a strictly increasing map $\psi: T \rightarrow T^{\prime}$ such that $\psi \circ h \leq h^{\prime}$.

Given strictly increasing surjections $h: S \rightarrow T$ and $h^{\prime}: S \rightarrow T^{\prime}$ satisfying these conditions, with totally ordered sets $T$ and $T^{\prime}$, there exists an isomorphism $\varphi: T \rightarrow T^{\prime}$ such that $h^{\prime}=\varphi \circ h$.

Proof. For $(1) \Rightarrow(2)$, suppose $\mathrm{ht}_{S}=\mathrm{ht}_{T} \circ \mathrm{h}$, and let $T^{\prime}$ be an ordered set, $h^{\prime}: S \rightarrow T^{\prime}$ strictly increasing. Then $h_{T^{\prime}} \circ h^{\prime}$ is strictly increasing, hence $\mathrm{ht}_{T} \circ h=\mathrm{ht}_{S} \leq \mathrm{ht}_{T^{\prime}} \circ h^{\prime}$, and $\operatorname{ht}(S)=\mathrm{ht}_{T}(h(S))$ is an initial segment of the range of $h_{T^{\prime}} \circ h^{\prime}$. We let $\iota$ denote the natural inclusion $h t(S) \hookrightarrow$ $\left(\mathrm{ht}_{T^{\prime}} \circ h^{\prime}\right)(S)$. For $y \in \mathrm{ht}\left(T^{\prime}\right)$ let $\mathrm{ht}_{T^{\prime}}^{-1}(y) \in T^{\prime}$ denote the smallest $x \in T^{\prime}$ such that ht $T_{T^{\prime}}(x)=y$. Then htt $T_{T^{\prime}}\left(\mathrm{ht}_{T^{\prime}}^{-1}(y)\right)=y$ and ht ${ }_{T^{\prime}}^{-1}\left(\mathrm{ht}_{T^{\prime}}(x)\right) \leq x$ for all $x \in T^{\prime}, y \in \mathrm{ht}\left(T^{\prime}\right)$. The map $\psi=\mathrm{ht}_{T^{\prime}}^{-1} \circ \iota \circ \mathrm{ht}_{T}$ is non-decreasing and satisfies $\psi(h(s)) \leq h^{\prime}(s)$ for all $s \in S$ as required.

The implication $(2) \Rightarrow(3)$ is trivial. Suppose that $h$ satisfies (3). Then for every strictly increasing function $h^{\prime}: S \rightarrow$ On there exists a strictly increasing map $\psi: T \rightarrow$ On such that $\psi \circ h \leq h^{\prime}$. Hence $\psi^{\prime}=\psi \circ \mathrm{ht}_{T}^{-1}$ is a strictly increasing embedding of the ordinal ht $(T)$ into On with $\psi^{\prime} \circ\left(\mathrm{ht}_{T} \circ h\right) \leq h^{\prime}$. Since $\psi^{\prime}(\alpha) \geq \alpha$ for all ordinals $\alpha<\operatorname{ht}(T)$, it follows that $\mathrm{ht}_{T} \circ h \leq h^{\prime}$. Hence $\mathrm{ht}_{T} \circ h=\mathrm{ht}_{S}$.

For the second part, let $T$ and $T^{\prime}$ be totally ordered sets, and let $h: S \rightarrow T$ and $h^{\prime}: S \rightarrow T^{\prime}$ be strictly increasing surjections satisfying these equivalent conditions. Then $\mathrm{ht}_{T} \circ h=\mathrm{ht}_{S}=\mathrm{ht}_{T^{\prime}} \circ h^{\prime}$, hence $h^{\prime}=\varphi \circ h$ for the isomor$\operatorname{phism} \varphi=\mathrm{ht}_{T^{\prime}}^{-1} \circ \mathrm{ht}_{T}: T \rightarrow T^{\prime}$.

Trees. A tree on a set $U$ is a non-empty final segment $T$ of $\left(U^{*}, \sqsupseteq\right)$. (Recall that $a \sqsupseteq b \Leftrightarrow b$ is a truncation of $a$.) The empty sequence $\varepsilon$ is the largest element of a tree $T$ on $U$, called the root of $T$. The elements of $T$ are called the nodes of the tree $T$, and the minimal elements of $T$ are called the leafs of $T$. Given a node $a=\left(a_{1}, \ldots, a_{n}\right)$ of $T$, we denote by $T_{a}$ the tree

$$
T_{a}:=\left\{b \in U^{*}: a b \in T\right\},
$$

called the subtree of $T$ with root at $a$. Let $S$ be a tree on $U$, and $T$ a tree on $V$. A map $\varphi: S \rightarrow T$ is called length-preserving if length $(\varphi(s))=\operatorname{length}(s)$ for 
all $s \in S$. Any increasing length-preserving map $S \rightarrow T$ is strictly increasing, and the image of $S$ is a tree on $V$. Moreover:

LEMMA 2.4. For a map $\varphi: S \rightarrow T$, the following are equivalent:

(1) $\varphi$ is increasing and length-preserving.

(2) $\varphi(\varepsilon)=\varepsilon$, and for all $s \in S, a \in U$ there exists $b \in V$ with $\varphi(s a)=$ $\varphi(s) b$.

Given a map $\varphi: U \rightarrow V$ we obtain an increasing length-preserving map $U^{*} \rightarrow V^{*}$, also denoted by $\varphi$, by setting

$$
\varphi\left(a_{1}, \ldots, a_{n}\right):=\left(\varphi\left(a_{1}\right), \ldots, \varphi\left(a_{n}\right)\right) \quad \text { for } a_{1}, \ldots, a_{n} \in U .
$$

Rank of a well-founded tree. Let $T$ be a well-founded tree on a set $U$. Then $\operatorname{ht}(T)=\operatorname{ht}(\varepsilon)+1$. (Recall that $\varepsilon$ is the root of $T)$. We call the ordinal ht $(\varepsilon)$ the $\operatorname{rank}$ of the tree $T$, denoted by $\operatorname{rk}(T)$. Hence $\operatorname{rk}(T)=0$ if and only if $T=\{\varepsilon\}$, and $\operatorname{ht}(a)=\operatorname{rk}\left(T_{a}\right)$ for any $a \in T$. Also note that

$$
\operatorname{rk}(T)=\sup \{\operatorname{ht}((x))+1: x \in U,(x) \in T\} .
$$

Every tree $T^{\prime}$ on $U$ with $T^{\prime} \subseteq T$ is well-founded with $\operatorname{rk}\left(T^{\prime}\right) \leq \operatorname{rk}(T)$. More generally:

Lemma 2.5. Let $S$ and $T$ be trees on $U$ and $V$, respectively. If $T$ is well-founded, then the following are equivalent:

(1) $S$ is well-founded with $\operatorname{rk}(S) \leq \operatorname{rk}(T)$.

(2) There exists a length-preserving increasing map $S \rightarrow T$.

(3) There exists a strictly increasing map $S \rightarrow T$.

Proof. For a proof of $(1) \Rightarrow(2)$ see, e.g., $[20,(2.9)]$. The implication $(2) \Rightarrow(3)$ is trivial, and $(3) \Rightarrow(1)$ follows from Lemma $2.2(1)$.

Invariants of Noetherian ordered sets. Every Noetherian ordered set $S$ is well-founded, hence has a certain height $\operatorname{ht}(S)$. Following [24], we now introduce two other ordinal-valued invariants associated to every Noetherian ordered set $S$, called the type and the width of $S$. Together, they measure the complexity of $S$. First, for an ordered set $S$ we define the following trees on $S$ :

$$
\begin{aligned}
& \operatorname{Dec}(S):=\left\{\left(s_{1}, \ldots, s_{n}\right) \in S^{*}: s_{i}>s_{j} \text { for all } 1 \leq i<j \leq n\right\}, \\
& \operatorname{Ant}(S):=\left\{\left(s_{1}, \ldots, s_{n}\right) \in S^{*}: s_{i} \| s_{j} \text { for all } 1 \leq i<j \leq n\right\}, \\
& \operatorname{Bad}(S):=\left\{\left(s_{1}, \ldots, s_{n}\right) \in S^{*}: s_{i} \not \leq s_{j} \text { for all } 1 \leq i<j \leq n\right\} .
\end{aligned}
$$

We call $\operatorname{Dec}(S)$ the tree of decreasing sequences of $S, \operatorname{Ant}(S)$ the tree of antichains of $S$, and $\operatorname{Bad}(S)$ the tree of bad sequences of $S$. Note that $S$ is wellfounded if and only if $\operatorname{Dec}(S)$ is well-founded, and $\operatorname{ht}(S)=\operatorname{rk}(\operatorname{Dec}(S))$. The tree $\operatorname{Ant}(S)$ is well-founded if and only if every antichain of $S$ is finite, and $S$ 
is Noetherian if and only if $\operatorname{Bad}(S)$ is well-founded. For any quasi-embedding $\varphi: S \rightarrow T$ of Noetherian ordered sets we have $\varphi(\operatorname{Ant}(S)) \subseteq \operatorname{Ant}(T)$ and $\varphi(\operatorname{Bad}(S)) \subseteq \operatorname{Bad}(T)$.

Definition 2.6. Let $S$ be a Noetherian ordered set. Then

(1) $\operatorname{wd}(S):=\operatorname{rk}(\operatorname{Ant}(S))$ is called the width of $S$, and

(2) o $(S):=\operatorname{rk}(\operatorname{Bad}(S))$ is called the type of $S$.

If the ordering on $S$ is total (i.e., $S$ is well-ordered) then $\operatorname{Dec}(S)=$ $\operatorname{Bad}(S)$, hence $\operatorname{rk}(\operatorname{Bad}(S))$ is the order type of $S$, justifying our choice of notation. Note that

$$
\operatorname{ht}_{\operatorname{Ant}(S)}(s)=\operatorname{wd}\left(S^{\| s}\right) \quad \text { for } s \in \operatorname{Ant}(S),
$$

where $S^{\| s}:=\left\{y \in S: y \| s_{i}\right.$ for all $\left.i\right\}$ for $s=\left(s_{1}, \ldots, s_{n}\right) \in S^{*}$, and

$$
\operatorname{ht}_{\operatorname{Bad}(S)}(s)=\mathrm{o}\left(S^{\nsupseteq s}\right) \quad \text { for } s \in \operatorname{Bad}(S),
$$

where $S^{\nsucceq s}:=\left\{y \in S: y \nsupseteq s_{i}\right.$ for all $\left.i\right\}$ for $s=\left(s_{1}, \ldots, s_{n}\right) \in S^{*}$.

Characterization of height and type. The height and the type of a Noetherian ordered set allow important reinterpretations. Recall that any total ordering extending a Noetherian ordering is a well-ordering (cf. Proposition 1.3).

TheOREM 2.7. Let $S$ be a Noetherian ordered set.

(1) There exists a total ordering on $S$ extending the given ordering of maximal possible order type; this order type equals $\mathrm{o}(S)$ (de JonghParikh [11]).

(2) There exists a chain of $S$ of maximal possible order type; this order type equals $\mathrm{ht}(S)$ (Wolk [47]).

Because of (1), the type of a Noetherian ordered set $S$ is sometimes also called the maximal order type of $S$. The width of $S$ is finite if and only if there is some $n$ such that every antichain in $S$ has size $\leq n$; the smallest such $n$ is $\operatorname{wd}(S)$. In this case, $S$ is a union of $\operatorname{wd}(S)$ chains (Dilworth [13]). In general (i.e., for infinite width), no characterization of the width similar to (1) or (2) in the theorem above seems to be known. (See [24, p. 77].) We refer to [1], [35] for discussions of Dilworth's Theorem in the case of infinite width.)

Basic facts about type and width. We record some basic properties:

Proposition 2.8. Let $S$ and $T$ be Noetherian ordered sets.

(1) o(S) $=\sup \left\{\mathrm{o}\left(S^{\nsucceq x}\right)+1: x \in S\right\}$.

(2) If there exists a quasi-embedding $S \rightarrow T$, then $\mathrm{o}(S) \leq \mathrm{o}(T)$. (In particular if $S \subseteq T$, then $\mathrm{o}(S) \leq \mathrm{o}(T)$.) 
(3) If there exists an increasing surjection $S \rightarrow T$, then $\mathrm{o}(S) \geq \mathrm{o}(T)$.

(4) $\mathrm{o}(S \amalg T)=\mathrm{o}(S) \oplus \mathrm{o}(T)$ and $\mathrm{o}(S \times T)=\mathrm{o}(S) \otimes \mathrm{o}(T)$.

Proof. Part (1) follows from (2.5) and (2.7), and part (2) from Lemma 2.2(1) and the remarks preceding Definition 2.6. For (3) suppose that $\varphi: S \rightarrow T$ is an increasing surjection. For every $t \in T$ choose $\psi(t) \in S$ with $\varphi(\psi(t))=t$. If $\left(t_{1}, \ldots, t_{n}\right) \in \operatorname{Bad}(T)$ then $\left(\psi\left(t_{1}\right), \ldots, \psi\left(t_{n}\right)\right) \in \operatorname{Bad}(S)$, so $\psi$ induces an increasing and length-preserving $\operatorname{map} \operatorname{Bad}(T) \rightarrow \operatorname{Bad}(S)$. Hence $\mathrm{o}(T)=\operatorname{rk}(\operatorname{Bad}(T)) \leq \operatorname{rk}(\operatorname{Bad}(S))=\mathrm{o}(S)$ by Lemma 2.2(1). For a proof of (4) see [11] or [24].

The computation of o $(S)$, for a concrete given Noetherian ordered set $S$, is often quite hard; see, e.g., [39]. In Section 4 we will bound the maximal order type of the Noetherian ordered set of monomial ideals.

Proposition 2.9. Let $S$ and $T$ be Noetherian ordered sets.

(1) $\operatorname{wd}(S)=\sup \left\{\mathrm{wd}\left(S^{\| x}\right)+1: x \in S\right\}$.

(2) If there exists a quasi-embedding $S \rightarrow T$, then $\operatorname{wd}(S) \leq \operatorname{wd}(T)$. (In particular if $S \subseteq T$, then $\operatorname{wd}(S) \leq \operatorname{wd}(T)$.

(3) If there exists an increasing surjection $S \rightarrow T$, then $\operatorname{wd}(S) \geq \operatorname{wd}(T)$.

(4) $\operatorname{wd}(S \amalg T)=\operatorname{wd}(S) \oplus \operatorname{wd}(T)$.

Proof. Part (1) follows from (2.5) and (2.6), and part (2) again from Lemma 2.2(1). The proof of (3) is similar to the proof of Proposition 2.8(3). Part (4) is shown by induction on $\mathrm{wd}(S) \oplus \mathrm{wd}(T)$ : Since $(S \amalg T)^{\| x}=S^{\| x} \amalg T$ if $x \in S$ and $(S \amalg T)^{\| x}=S \amalg T^{\| x}$ if $x \in T$, from (1) we get

$$
\operatorname{wd}(S \amalg T)=\sup \left\{\operatorname{wd}\left(S^{\| x} \amalg T\right)+1, \operatorname{wd}\left(S \amalg T^{\| y}\right)+1: x \in S, y \in T\right\} .
$$

By inductive hypothesis and (2.3) we get $\operatorname{wd}(S \amalg T)=\operatorname{wd}(S) \oplus \operatorname{wd}(T)$.

A formula for the width of $\alpha \times \beta$ ordered componentwise, where $\alpha$ and $\beta$ are ordinals, can be found in [1], and a formula for the width of $S \times T$ ordered lexicographically, for Noetherian ordered sets $S$ and $T$, in [2].

Connections between the invariants. Height, width and type are related by the following inequality:

Proposition 2.10 (Height-Width Theorem, [24]). Let $S$ be a Noetherian ordered set. Then

$$
\mathrm{o}(S) \leq \mathrm{ht}(S) \otimes \mathrm{wd}(S)
$$

This generalizes the well-known fact that a finite ordered set with at least $r s+1$ elements contains a chain with $r+1$ elements or an antichain with $s+1$ elements.

Proof. Since we will need a similar idea below (Lemma 2.19), we sketch the proof of Proposition 2.10. Let $g=h_{\operatorname{Ant}(S)}$ be the height function of the 
tree of antichains of $S$, and define $h: \operatorname{Bad}(S) \backslash\{\varepsilon\} \rightarrow \operatorname{wd}(S)$ by

$$
\begin{aligned}
& \left(s_{1}, \ldots, s_{n}\right):= \\
& \quad \min \left\{g\left(s_{i_{1}}, \ldots, s_{i_{m}}\right): 1 \leq i_{1}<\cdots<i_{m}=n, \operatorname{ht}\left(s_{i_{1}}\right) \leq \cdots \leq \operatorname{ht}\left(s_{i_{m}}\right)\right\}
\end{aligned}
$$

for $\left(s_{1}, \ldots, s_{n}\right) \in \operatorname{Bad}(S), n \geq 1$. It is easy to see that $f(\varepsilon):=\varepsilon$ and

$$
\begin{aligned}
f\left(s_{1}, \ldots, s_{n}\right) & := \\
& \left(\left(\operatorname{ht}\left(s_{1}\right), h\left(s_{1}\right)\right),\left(\mathrm{ht}\left(s_{2}\right), h\left(s_{1}, s_{2}\right)\right), \ldots,\left(\operatorname{ht}\left(s_{n}\right), h\left(s_{1}, \ldots, s_{n}\right)\right)\right)
\end{aligned}
$$

defines a strictly increasing map $f: \operatorname{Bad}(S) \rightarrow \operatorname{Bad}(\operatorname{ht}(S) \times \operatorname{wd}(S))$. Hence

$$
\mathrm{o}(S) \leq \mathrm{o}(\mathrm{ht}(S) \times \operatorname{wd}(S))=\operatorname{ht}(S) \otimes \operatorname{wd}(S)
$$

by Lemma 2.5 and Proposition 2.8(4).

The following proposition connects the type of a Noetherian ordered set $S$ with the height of the well-founded ordered set $(\mathcal{F}(S), \supseteq)$ of its final segments:

Proposition 2.11 (Bonnet-Pouzet [7]). For every Noetherian ordered set $S$,

$$
\operatorname{ht}(\mathcal{F}(S))=\mathrm{o}(S)+1
$$

Let us outline the main idea of the proof of this fact. First we note that Proposition 2.11 is a consequence of the following lemma, the characterization (2.4) of the height, Theorem 2.7(1), and the fact that ht $(\mathcal{F}(\alpha))=\alpha+1$ for any ordinal $\alpha$.

Lemma 2.12. Let $(S, \leq)$ be an ordered set. The assignment $\leq^{\prime} \mapsto \mathcal{F}\left(S, \leq^{\prime}\right)$ is a one-to-one correspondence between the total orderings $\leq$ on $S$ extending $\leq$ and the maximal chains of the ordered $\operatorname{set}(\mathcal{F}(S, \leq), \supseteq)$.

It is easy to verify that the map in the lemma is well-defined, and it is clearly one-to-one. Now let $\mathcal{C}$ be a maximal chain of $\mathcal{F}(S, \leq)$. Define a binary relation $\leq_{\mathcal{C}}$ on $S$ by $x \leq_{\mathcal{C}} y: \Leftrightarrow$ every $F \in \mathcal{C}$ which contains $x$ also contains $y$. The main work consists in establishing that for any two distinct elements $x \neq y$ of $S$ for which there exists $F \in \mathcal{F}(S, \leq)$ with $x \in F, y \notin F$, there exists $G \in \mathcal{C}$ with $x \in G, y \notin G$. (For the details see [7].) From this it is straightforward to check that $\leq_{\mathcal{C}}$ is a total ordering on $S$ extending $\leq$, and $\mathcal{F}\left(S, \leq_{\mathcal{C}}\right)=\mathcal{C}$.

Height and minimal order type. Let $(S, \leq)$ be a Noetherian ordered set. Then there is a smallest ordinal $\alpha$ such that $\leq$ has an extension to a well-ordering on $S$ of order type $\alpha$. We call $\alpha$ the minimal order type of $S$, denoted by o* $(S)$. We show here that this ordinal agrees with the height of $S$ if $\operatorname{ht}(S)$ is a limit ordinal, and differs from the height of $S$ at most by a finite ordinal otherwise. (This was observed in [39, pp. 8-10].) In the first 
case we also show how to obtain an extension $\leq^{*}$ of $\leq$ to a well-ordering of $S$ of order type o $^{*}(S)$. This will all be based on the following observation:

LEMMA 2.13. The height function ht: $S \rightarrow \mathrm{ht}(S)$ has finite fibers.

Proof. By Lemma 1.5(1) and the fact that ht is strictly increasing.

Let now $\leq^{\prime}$ be any extension of $\leq$ to a well-ordering on $S$. Define a binary relation $\leq^{*}$ on $S$ by

$$
x \leq^{*} y: \Leftrightarrow(\operatorname{ht}(x), x) \leq_{\operatorname{lex}}(\operatorname{ht}(y), y) .
$$

Here $\leq_{\text {lex }}$ denotes the lexicographic product of the ordering of $\operatorname{ht}(S)$ and $\leq^{\prime}$, that is,

$$
(\alpha, x) \leq_{\text {lex }}(\beta, y) \Leftrightarrow \alpha<\beta \text { or }\left(\alpha=\beta \text { and } x \leq^{\prime} y\right)
$$

for all $\alpha, \beta<\operatorname{ht}(S)$ and $x, y \in S$. It is straightforward to check that $\leq^{*}$ is an extension of $\leq$ to a well-ordering of $S$. We denote the height function of $\left(S, \leq^{*}\right)$ by $\mathrm{ht}^{*}: S \rightarrow \mathrm{ht}^{*}(S)$.

Lemma 2.14. For all $x \in S$, ht* $(x)<\mathrm{ht}(x)+\omega$.

Proof. By transfinite induction on $\alpha=\operatorname{ht}(x)$. If $\alpha=0$, then $x$ is one of the finitely many minimal elements of $(S, \leq)$. Hence there are only finitely many $y \in S$ with $y \leq^{*} x$, hence ht ${ }^{*}(x)<\omega$. For the successor case, suppose that $\operatorname{ht}(x)=\alpha+1$, and choose $y<x$ with ht $(y)=\alpha$. There are only finitely many elements $y=y_{0}<^{*} y_{1}<^{*} \cdots<^{*} y_{m}=x$ of $S$ which lie between $y$ and $x$ in the ordering $\leq^{*}$. So $\mathrm{ht}^{*}(x)=\mathrm{ht}^{*}(y)+m$, and by induction we get ht ${ }^{*}(y)<\mathrm{ht}(y)+\omega=\alpha+\omega$. Hence ht* $(x)<(\alpha+1)+\omega=\operatorname{ht}(x)+\omega$. Now suppose that $\alpha$ is a limit ordinal. Let $x_{0}<^{*} x_{1}<^{*} \cdots<^{*} x_{n}=x$ be the elements of height $\alpha$ which are $\leq^{*} x$; so ht* $(x)=$ ht $^{*}\left(x_{0}\right)+n$. We have ht $(y)<\operatorname{ht}(x)$ for all $y \in S$ with $y<^{*} x_{0}$, hence ht* $(y)<\operatorname{ht}(y)+\omega \leq \operatorname{ht}(x)$ by inductive hypothesis and since $\mathrm{ht}(x)=\alpha$ is a limit ordinal. Therefore

$$
\mathrm{ht}^{*}\left(x_{0}\right)=\sup \left\{\mathrm{ht}^{*}(y)+1: y<^{*} x_{0}\right\} \leq \mathrm{ht}(x),
$$

and hence ht* $(x)<\operatorname{ht}(x)+\omega$ as required.

Corollary 2.15. (1) If $\mathrm{ht}(S, \leq)$ is a successor ordinal, then

$$
\mathrm{ht}(S, \leq) \leq \mathrm{o}^{*}(S, \leq) \leq \mathrm{o}\left(S, \leq^{*}\right)<\mathrm{ht}(S, \leq)+\omega .
$$

(2) If $\mathrm{ht}(S, \leq)$ is a limit ordinal, then

$$
\mathrm{o}^{*}(S, \leq)=\mathrm{o}\left(S, \leq^{*}\right)=\operatorname{ht}(S, \leq) \text {. }
$$

In Section 4 we will apply this corollary in the following situation: Suppose that $S$ is a Noetherian ordered set with a largest element $s_{0}$ whose height ht $\left(s_{0}\right)$ is a limit ordinal. Then the Noetherian ordered set $S_{0}=S \backslash\left\{s_{0}\right\}$ has height $\mathrm{ht}\left(s_{0}\right)$. By part $(1)$ of the corollary, it follows that o* $\left(S_{0}\right)=\operatorname{ht}\left(s_{0}\right)$ and hence $S$ has minimal order type o* $(S)=\operatorname{ht}\left(s_{0}\right)+1=\operatorname{ht}(S)$. 
Total orderings of monomials. As an illustration for the material in this section, we now compute the invariants ht, o, wd, and ht* for the Noetherian ordered set $\mathbb{N}^{m}$, and hence for the set of monomials in the polynomial ring $K\left[X_{1}, \ldots, X_{m}\right]$ over a field $K$, ordered by divisibility (see Example 1.1). It is convenient to consider, slightly more generally, Noetherian ordered sets of the form $\mathbb{N}^{m} \times S$, where $S$ is a finite non-empty ordered set.

Lemma 2.16. Let $S$ be a finite non-empty ordered set and $m>0$. Then

$$
\operatorname{ht}\left(\mathbb{N}^{m} \times S\right)=\omega, \quad \mathrm{o}\left(\mathbb{N}^{m} \times S\right)=\omega^{m}|S|, \quad \mathrm{ht}^{*}\left(\mathbb{N}^{m} \times S\right)=\omega .
$$

Proof. The function $(\nu, s) \mapsto|\nu|+\mathrm{ht}_{S}(s): \mathbb{N}^{m} \times S \rightarrow \mathbb{N}$ is strictly increasing. Hence $\operatorname{ht}((\nu, s))=|\nu|+\mathrm{ht}_{S}(s)$ for all $(\nu, s) \in \mathbb{N}^{m} \times S$, and ht $\left(\mathbb{N}^{m} \times S\right)=\omega$. By Corollary 2.15(2) this yields ht* $\left(\mathbb{N}^{m} \times S\right)=\omega$. By Proposition 2.8(4) we get $\mathrm{o}\left(\mathbb{N}^{m} \times S\right)=\mathrm{o}\left(\mathbb{N}^{m}\right) \otimes \mathrm{o}(S)=\omega^{m}|S|$.

The lexicographic ordering $\leq_{\text {lex }}$ on $\mathbb{N}^{m}$ is an example for a total ordering of $\mathbb{N}^{m}$ extending the product ordering $\leq$ and having maximal order type. Given any total ordering $\leq^{\prime}$ on $\mathbb{N}^{m}$ extending $\leq$, we obtain a total ordering $\leq^{*}$ on $\mathbb{N}^{m}$ of minimal order type $\omega$ extending $\leq$, as shown in (2.8):

$$
\nu \leq^{*} \mu: \Leftrightarrow|\nu|<|\mu| \text {, or }|\nu|=|\mu| \text { and } \nu \leq^{\prime} \mu .
$$

For $\leq^{\prime}=\leq_{\text {lex }}$ (the lexicographic ordering of $\mathbb{N}^{m}$ ) the ordering obtained in this way is commonly called the degree-lexicographic ordering of $\mathbb{N}^{m}$. Orderings of the form $\leq^{*}$ are called degree-compatible. In applications, one is usually interested in total orderings $\leq^{\prime}$ extending $\leq$ which are semigroup orderings, that is, which satisfy the condition

$$
\nu \leq^{\prime} \mu \Rightarrow \nu+\lambda \leq^{\prime} \mu+\lambda \quad \text { for all } \nu, \mu, \lambda \in \mathbb{N}^{m} .
$$

A total semigroup ordering on $\mathbb{N}^{m}$ extending $\leq$ is called a term ordering. Via the usual identification of $\mathbb{N}^{m}$ with $X^{\diamond}$, term orderings on $\mathbb{N}^{m}$ hence correspond to term orderings on $X^{\diamond}$ (as defined in Remark 1.10), where $X=$ $\left\{X_{1}, \ldots, X_{m}\right\}$ carries the trivial ordering. The lexicographic and degreelexicographic orderings of $\mathbb{N}^{m}$ are term orderings. A complete description of all term orderings on $\mathbb{N}^{m}$ is available [37]: For any such ordering $\leq^{\prime}$ there exists an invertible $m \times m$-matrix $A$ with real coefficients such that

$$
\nu \leq^{\prime} \mu \Leftrightarrow A \nu \leq_{\text {lex }} A \mu \quad \text { for all } \nu, \mu \in \mathbb{N}^{m},
$$

where $\leq_{\text {lex }}$ denotes the lexicographic ordering on $\mathbb{R}^{m}$. Conversely, any matrix $A \in \mathrm{GL}(m, \mathbb{R})$ satisfying $A e_{i} \geq_{\text {lex }} 0$ for all $i=1, \ldots, m$ (where $e_{i}$ denotes the $i$ th unit vector in $\mathbb{R}^{m}$ ) gives rise to a term ordering $\leq^{\prime}$ on $\mathbb{N}^{m}$, via (2.9). In particular, the order types of term orders on $\mathbb{N}^{m}$ are the ordinals of the form $\omega^{k}$ with $1 \leq k \leq m$. There are only $m$ ! different term orderings of maximal order type $\omega^{m}$ on $\mathbb{N}^{m}$ (obtained by choosing permutation matrices for $A$ ), and for each $1 \leq k<m$ there are continuum many term orderings on $\mathbb{N}^{m}$ with order type $\omega^{k}$. (See [29].) 
More generally, a ranking of $\mathbb{N}^{m} \times S$, where $S$ is a finite non-empty set, is a total ordering of $\mathbb{N}^{m} \times S$ which extends the product ordering on $\mathbb{N}^{m} \times S$ (where $S$ is equipped with the trivial ordering) and satisfies

$$
(\nu, s) \leq(\mu, t) \Rightarrow(\nu+\lambda, s) \leq(\mu+\lambda, t) \quad \text { for all } \nu, \mu, \lambda \in \mathbb{N}^{m} .
$$

Rankings play a role in algorithmic differential algebra (e.g., in the theory of Riquier-Janet bases) similar to the role played by term orderings in ordinary algorithmic algebra (in the theory of Gröbner bases), see [22], [38]: the elements $(\nu, i)$ of $\mathbb{N}^{m} \times\{1, \ldots, n\}$ correspond to the derivatives $\partial^{|\nu|} y^{i} / \partial X_{1}^{\nu_{1}} \cdots \partial X_{m}^{\nu_{m}}$, where $y^{1}, \ldots, y^{n}$ are differential indeterminates over a differential ring with $m$ commuting derivations $\partial / \partial X_{1}, \ldots, \partial / \partial X_{m}$. Rankings also naturally arise when Gröbner basis theory is generalized to finitely generated free modules over $K[X]$ (see [15, Chapter 15]). We refer to [38] for a (rather involved) classification of rankings which extends the one of term orderings described above. It would be interesting to determine the possible order types of rankings from this classification.

We now turn to $\operatorname{wd}\left(\mathbb{N}^{m} \times S\right)$. By the Height-Width Theorem 2.10 we have $\operatorname{wd}\left(\mathbb{N}^{m} \times S\right) \geq \omega^{m-1}|S|$, since ht $\left(\mathbb{N}^{m} \times S\right)=\omega, \mathrm{o}\left(\mathbb{N}^{m} \times S\right)=\omega^{m}|S|$. We will show:

Proposition 2.17. $\operatorname{wd}\left(\mathbb{N}^{m} \times S\right)=\omega^{m-1}|S|$ for all $m>0$ and all finite non-empty ordered sets $S$.

In the proof, we will use the following lemma.

Lemma 2.18. Let $T_{1}, \ldots, T_{m}$ be well-ordered sets, $T=T_{1} \times \cdots \times T_{m}$. Then, for any $a=\left(a_{1}, \ldots, a_{m}\right) \in T$,

$$
\operatorname{wd}\left(T^{\| a}\right)=\bigoplus_{\varepsilon} \operatorname{wd}\left(T_{1}^{\varepsilon_{1} a_{1}} \times \cdots \times T_{m}^{\varepsilon_{n} a_{m}}\right),
$$

where the sum runs over all $\varepsilon=\left(\varepsilon_{1}, \ldots, \varepsilon_{m}\right) \in\{\leq,>\}^{m}$ such that for some $i, j$, we have $\varepsilon_{i}=\leq$ and $\varepsilon_{j}=>$.

Proof. By (2.6), the fact that

$$
T^{\| a}=\coprod_{\varepsilon} T_{1}^{\varepsilon_{1} a_{1}} \times \cdots \times T_{m}^{\varepsilon_{m} a_{m}},
$$

and Proposition 2.9(4).

In order to prove Proposition 2.17 , it suffices to show $\operatorname{wd}\left(\mathbb{N}^{m} \times S\right) \leq$ $\omega^{m-1}|S|$ for all $m>0$ and finite ordered sets $S \neq \emptyset$. We proceed by induction on $m$. Note first that if $M \neq \emptyset$ is an ordered set, then we have a natural quasiembedding of $M \times S$ into $M \amalg \cdots \amalg M(|S|$ times); hence if $M$ is Noetherian, then $\operatorname{wd}(M \times S) \leq \operatorname{wd}(M)|S|$. For $M=\mathbb{N}$ this yields $\operatorname{wd}(\mathbb{N} \times S) \leq|S|$, and hence the base case $m=1$ of our induction. Now suppose $m>1$. With 
$T=\mathbb{N}^{m}$, we have

$$
\operatorname{wd}(T)=\sup \left\{\operatorname{wd}\left(T^{\| a}\right)+1: a \in T\right\} .
$$

Let $\varepsilon=\left(\varepsilon_{1}, \ldots, \varepsilon_{m}\right) \in\{\leq,>\}^{m}$ be such that for some $i, j$, we have $\varepsilon_{i}=\leq$ and $\varepsilon_{j}=>$, and let $a=\left(a_{1}, \ldots, a_{m}\right) \in \mathbb{N}^{m}$. For $b \in \mathbb{N}$, if $\varepsilon=\leq$, then $\mathbb{N}^{\varepsilon b}$ is finite, and if $\varepsilon=>$, then $\mathbb{N}^{\varepsilon b} \cong \mathbb{N}$. It follows that $\mathbb{N}^{\varepsilon_{1} a_{1}} \times \cdots \times \mathbb{N}^{\varepsilon_{n} a_{m}} \cong$ $\mathbb{N}^{k} \times U$ for some $1 \leq k<m$ and some non-empty finite ordered set $U$. By induction $\operatorname{wd}\left(\mathbb{N}^{\varepsilon_{1} a_{1}} \times \cdots \times \mathbb{N}^{\varepsilon_{n} a_{m}}\right) \leq \omega^{k-1}|U|$. By the lemma, this yields $\operatorname{wd}\left(\left(\mathbb{N}^{m}\right)^{\| a}\right)<\omega^{m-1}$ and thus $\operatorname{wd}\left(\mathbb{N}^{m} \times S\right) \leq \operatorname{wd}\left(\mathbb{N}^{m}\right)|S| \leq \omega^{m-1}|S|$ as desired.

A variant of the tree of antichains. Let $S$ be a Noetherian ordered set. In Section 4, we will need a variant of the tree of antichains $\operatorname{Ant}(S)$ of $S$. For this, we fix a total ordering $\leq$ extending the ordering $\leq$ of $S$. We define a tree

$$
\operatorname{Ant}_{\leq^{\prime}}(S):=\left\{\left(s_{1}, \ldots, s_{n}\right) \in S^{*}: s_{i} \| s_{j} \text { and } s_{i}<^{\prime} s_{j} \text { for all } 1 \leq i<j \leq n\right\}
$$

on $S$, an ordered subset of the tree $\operatorname{Ant}(S)$ of antichains of $S$. We clearly have $\operatorname{rk}\left(\operatorname{Ant}_{\leq^{\prime}}(S)\right) \leq \operatorname{rk}(\operatorname{Ant}(S))=\operatorname{wd}(S)$, and we conjecture that in general, the reverse inequality is also true. Here, we confine ourselves to showing:

LEMMA 2.19. $\operatorname{rk}\left(\operatorname{Ant}_{\leq^{\prime}}\left(\mathbb{N}^{m} \times S\right)\right)=\omega^{m-1}|S|$ for any finite non-empty ordered set $S$ and total ordering $\leq 1$ on $\mathbb{N}^{m} \times S$ of order type $\omega$ extending the product ordering.

This follows immediately from Proposition 2.17 above and the following fact:

Lemma 2.20. o $(S) \leq \mathrm{o}\left(S, \leq^{\prime}\right) \otimes \operatorname{rk}\left(\operatorname{Ant}_{\leq^{\prime}}(S)\right)$ for any Noetherian ordered set $(S, \leq)$ and any total ordering $\leq$ extending $\leq$.

Proof. Put $\alpha=\operatorname{rk}\left(\operatorname{Ant}_{\leq^{\prime}}(S)\right)$. Let $g=\operatorname{ht}_{\mathrm{Ant}_{\leq^{\prime}}}(S)$ be the height function of $\operatorname{Ant}_{\leq^{\prime}}(S)$. Define $h: \operatorname{Bad}(S) \backslash\{\varepsilon\} \rightarrow \alpha$ by

$$
\begin{aligned}
& h\left(s_{1}, \ldots, s_{n}\right) \\
& \quad:=\min \left\{g\left(s_{i_{1}}, \ldots, s_{i_{m}}\right): 1 \leq i_{1}<\cdots<i_{m}=n, s_{i_{1}}<\cdots<{ }^{\prime} s_{i_{m}}\right\},
\end{aligned}
$$

for $\left(s_{1}, \ldots, s_{n}\right) \in \operatorname{Bad}(S), n \geq 1$. Then $f(\varepsilon):=\varepsilon$ and

$$
f\left(s_{1}, \ldots, s_{n}\right):=\left(\left(s_{1}, h\left(s_{1}\right)\right),\left(s_{1}, h\left(s_{1}, s_{2}\right)\right), \ldots,\left(s_{n}, h\left(s_{1}, \ldots, s_{n}\right)\right)\right)
$$

defines a strictly increasing map

$$
f: \operatorname{Bad}(S) \rightarrow \operatorname{Bad}\left(\left(S, \leq^{\prime}\right) \times \alpha\right) .
$$

Hence $\mathrm{o}(S) \leq \mathrm{o}\left(\left(S, \leq^{\prime}\right) \times \alpha\right)=\mathrm{o}\left(S, \leq^{\prime}\right) \otimes \alpha$. 


\section{THE ORDERED SET OF HILBERT POLYNOMIALS}

In this section we discuss the sets of Hilbert and Hilbert-Samuel polynomials of finitely generated graded $K$-algebras (where $K$ is a field) as ordered sets, with the ordering given by the relation of eventual dominance. Macaulay's Theorem on the possible Hilbert functions of such $K$-algebras will play an important role. We begin by recalling this theorem and some of its consequences, in particular a description of all Hilbert-Samuel polynomials of finitely generated graded $K$-algebras. This description will be used to give an interpretation of the height function on $\mathcal{F}\left(\mathbb{N}^{m}\right)$ in terms of the coefficients of the Hilbert-Samuel polynomial. We give two applications concerning increasing chains of ideals in polynomial rings.

Integer-valued polynomials. Recall that a polynomial $f(T) \in \mathbb{Q}[T]$ (in a single variable $T$ ) is called an integer-valued polynomial if $f(s)$ is an integer for all $s \in \mathbb{N}$. For example,

$$
\left(\begin{array}{l}
T \\
j
\end{array}\right)=\frac{T(T-1) \cdots(T-j+1)}{j !} \in \mathbb{Q}[T] \quad(j \in \mathbb{N})
$$

is integer-valued. The polynomials $\left(\begin{array}{c}T+i \\ i\end{array}\right)$ (for $i \in \mathbb{N}$ ) form a basis for the $\mathbb{Z}$-submodule of $\mathbb{Q}[T]$ consisting of the integer-valued polynomials, i.e., every non-zero integer-valued polynomial $f(T) \in \mathbb{Q}[T]$ can be uniquely written in the form

$$
f(T)=b_{d}\left(\begin{array}{c}
T+d \\
d
\end{array}\right)+b_{d-1}\left(\begin{array}{c}
T+d-1 \\
d-1
\end{array}\right)+\cdots+b_{0}\left(\begin{array}{c}
T+0 \\
0
\end{array}\right)
$$

with $b_{0}, \ldots, b_{d} \in \mathbb{Z}, b_{d} \neq 0$. We totally order the integer-valued polynomials by dominance: if $f(T)=\sum_{i=0}^{d} b_{i}\left(\begin{array}{c}T+i \\ i\end{array}\right)$ and $g(T)=\sum_{j=0}^{d} c_{j}\left(\begin{array}{c}T+j \\ j\end{array}\right)$ with $b_{i}, c_{j} \in \mathbb{Z}$, then

$$
\begin{aligned}
f(T) \preceq g(T) & : \Leftrightarrow f(s) \leq g(s) \text { for all } s \gg 0 \\
& \Leftrightarrow\left(b_{d}, b_{d-1}, \ldots, b_{0}\right) \leq_{\text {lex }}\left(c_{d}, c_{d-1}, \ldots, c_{0}\right) \text { in } \mathbb{Z}^{d+1} .
\end{aligned}
$$

With this ordering, the ring of integer-valued polynomials becomes an ordered integral domain.

Hilbert polynomials of homogeneous ideals. In this section, $K$ denotes a field. Let $I$ be a homogeneous ideal of a polynomial ring $K[X]=$ $K\left[X_{1}, \ldots, X_{m}\right]$ over $K$; that is, the ideal $I$ is generated by homogeneous elements of positive degree. Then as a $K$-vector space, $R=K[X] / I$ has a direct-sum decomposition $R=R_{0} \oplus R_{1} \oplus \cdots$ given by

$$
R_{s}:=\{f+I \in R: f \in K[X] \text { has total degree } s\} .
$$

This decomposition makes $R$ into a graded $K$-algebra: we have $R_{0}=K$ and $R_{s} \cdot R_{t} \subseteq R_{s+t}$ for all $s, t$. Each component $R_{s}$ is a finite-dimensional vector 
space over $K$. The function $H_{I}: \mathbb{N} \rightarrow \mathbb{N}$ defined by $H_{I}(s)=\operatorname{dim}_{K} R_{s}$ is called the Hilbert function of $I$. There exists an integer-valued polynomial $P_{I}$ of degree $<m$ (the Hilbert polynomial of $I$ ) such that $s \mapsto P_{I}(s)$ agrees with $s \mapsto H_{I}(s)$ for sufficiently large $s$. The degree of $P_{I}$ is one less than the Krull dimension of the ring $R$. (See, e.g., [15, Corollary 13.7].) If $I=(1)$ is the unit ideal in $K[X]$, we put $H_{I}(s)=0$ for all $s$ and $P_{I}=0$. As usual the degree of the zero polynomial is $\operatorname{deg} 0:=-1$.

For a final segment $E$ of $\mathbb{N}^{m}$, we call $H_{E}:=H_{I_{E}}$ and $P_{E}:=P_{I_{E}}$ the Hilbert function and Hilbert polynomial of $E$, respectively, where $I_{E} \subseteq \mathbb{Q}[X]$ is the monomial ideal corresponding to $E$. Given any final segment $E$ of $\mathbb{N}^{m}$, let us write $V_{E}:=\mathbb{N}^{m} \backslash E$ for the complement of $E$ in $\mathbb{N}^{m}$ (an initial segment of $\left.\mathbb{N}^{m}\right)$. We then have $H_{E}(n)=\left|V_{E, n}\right|$ for all $n$, where $Z_{n}:=\{z \in Z$ : $|z|=n\}$ for $Z \subseteq \mathbb{N}^{m}$.

Macaulay's Theorem. A classical theorem of Macaulay characterizes those functions $f: \mathbb{N} \rightarrow \mathbb{N}$ which arise as Hilbert functions of homogeneous ideals $I \subseteq K[X]$. Before we state Macaulay's Theorem, we have to introduce some more notation. (As a general reference for this material, we recommend [10, Chapter 4].) Given an integer $d \geq 1$, every positive integer $a$ can be written uniquely in the form

$$
a=\left(\begin{array}{c}
a_{d} \\
d
\end{array}\right)+\left(\begin{array}{c}
a_{d-1} \\
d-1
\end{array}\right)+\cdots+\left(\begin{array}{c}
a_{1} \\
1
\end{array}\right)
$$

where $a_{d}>a_{d-1}>\cdots>a_{1} \geq 0$. This sum is called the $d$ th Macaulay representation of $a$, and $\left(a_{d}, \ldots, a_{1}\right)$ are called the $d$ th Macaulay coefficients of $a$. We have $a \leq b$ if and only if $\left(a_{d}, \ldots, a_{1}\right) \leq_{\text {lex }}\left(b_{d}, \ldots, b_{1}\right)$. We define

$$
a^{\langle d\rangle}=\left(\begin{array}{c}
a_{d}+1 \\
d+1
\end{array}\right)+\left(\begin{array}{c}
a_{d-1}+1 \\
(d-1)+1
\end{array}\right)+\cdots+\left(\begin{array}{c}
a_{1}+1 \\
1+1
\end{array}\right)
$$

and $0^{\langle d\rangle}:=0$. We have (for a proof see $[10$, p. 162]):

Theorem 3.1 (Macaulay, [27]). Let $f: \mathbb{N} \rightarrow \mathbb{N}$. The following are equivalent:

(1) There exists a homogeneous ideal $I \subseteq K[X]$ with $H_{I}=f$.

(2) We have $f(1)=m$, and if $M_{n}$ denotes the set of the first $f(n)$ elements of $\mathbb{N}^{m}$ of degree $n$, in the lexicographic ordering, then $M=$ $\bigcup_{n \in \mathbb{N}} M_{n}$ is an initial segment of $\mathbb{N}^{m}$.

(3) $f(0)=1, f(1)=m$, and $f(n+1) \leq f(n)^{\langle n\rangle}$ for all $n \geq 1$.

A final segment $E$ of $\mathbb{N}^{m}$ is called a lex-segment of $\mathbb{N}^{m}$ if for every $n$ the set $E_{n}=\{e \in E:|e|=n\}$ of elements of $E$ having degree $n$ is a final segment of $\left(\mathbb{N}^{m}\right)_{n}$ under the lexicographic ordering. (This terminology is used slightly differently in [10].) If $M$ is as in statement (2) of the theorem, then clearly $\mathbb{N}^{m} \backslash M$ is a lex-segment of $\mathbb{N}^{m}$ with Hilbert function $f$. 
The zero ideal (0) of $K[X]=K\left[X_{1}, \ldots, X_{m}\right]$ has Hilbert polynomial $\left(\begin{array}{c}T+m-1 \\ m-1\end{array}\right)$. The following characterization of Hilbert polynomials of non-zero ideals is well-known:

Corollary 3.2. A polynomial $P(T) \in \mathbb{Q}[T]$ is a Hilbert polynomial of some non-zero homogeneous ideal of $K[X]=K\left[X_{1}, \ldots, X_{m}\right]$ if and only if

$$
P(T)=\left(\begin{array}{c}
T+a_{1} \\
a_{1}
\end{array}\right)+\left(\begin{array}{c}
T+a_{2}-1 \\
a_{2}
\end{array}\right)+\cdots+\left(\begin{array}{c}
T+a_{s}-(s-1) \\
a_{s}
\end{array}\right)
$$

for certain integers $m-1>a_{1} \geq a_{2} \geq \cdots \geq a_{s} \geq 0$, with $s \geq 1$.

Proof. Let $I \subseteq K[X], I \neq(0)$, be a homogeneous ideal with Hilbert function $f=H_{I}$ and Hilbert polynomial $P$. Macaulay's Theorem implies (see [10, Corollary 4.2.14]) that there exists an integer $n_{0} \in \mathbb{N}$ such that $f(n+1)=f(n)^{\langle n\rangle}$ for all $n \geq n_{0}$. We have $f(n)=P(n)$ for all $n \geq n_{0}$ : Let

$$
f\left(n_{0}\right)=\left(\begin{array}{c}
c_{n_{0}} \\
n_{0}
\end{array}\right)+\left(\begin{array}{c}
c_{n_{0}-1} \\
n_{0}-1
\end{array}\right)+\cdots+\left(\begin{array}{c}
c_{1} \\
1
\end{array}\right)
$$

be the $n_{0}$ th Macaulay representation of $f\left(n_{0}\right)$, and $j \geq 1$ minimal with $c_{j} \geq j$, so $c_{n_{0}}>c_{n_{0}-1}>\cdots>c_{j} \geq j \geq 1$. Then for $n=n_{0}+k$ with $k \geq 0$,

$$
\begin{aligned}
f(n) & =\left(\begin{array}{c}
c_{n_{0}}+k \\
n_{0}+k
\end{array}\right)+\left(\begin{array}{c}
c_{n_{0}-1}+k \\
n_{0}-1+k
\end{array}\right)+\cdots+\left(\begin{array}{c}
c_{j}+k \\
j+k
\end{array}\right) \\
& =\left(\begin{array}{c}
n+a_{1} \\
a_{1}
\end{array}\right)+\left(\begin{array}{c}
n+a_{2}-1 \\
a_{2}
\end{array}\right)+\cdots+\left(\begin{array}{c}
n+a_{s}-(s-1) \\
a_{s}
\end{array}\right),
\end{aligned}
$$

where $a_{n_{0}-i+1}=c_{i}-i$ for $i=j, \ldots, n_{0}$, and $s=n_{0}-j+1>0$. We have $a_{1}=\operatorname{deg} P=\operatorname{dim} I-1<m-1$, and hence $m-1>a_{1} \geq \cdots \geq a_{s} \geq 0$. Conversely, suppose $P(T)$ is an integer-valued polynomial in the form given in the corollary; we may assume $P \neq 0$. For $n \geq s$,

$$
P(n)=\left(\begin{array}{c}
n+a_{1} \\
n
\end{array}\right)+\left(\begin{array}{c}
n+a_{2}-1 \\
n-1
\end{array}\right)+\cdots+\left(\begin{array}{c}
n+a_{s}-(s-1) \\
n-(s-1)
\end{array}\right)
$$

with $n+a_{1}>n+a_{2}-1>\cdots>n+a_{s}-(s-1)>0$ is the $n$th Macaulay representation of $P(n)$. Hence $P(n+1)=P(n)^{\langle n\rangle}$ for all $n \geq s$. The $n$th Macaulay coefficients of $\left(\begin{array}{c}n+m-1 \\ n\end{array}\right)$ are $(n+m-1,0, \ldots, 0)$. Since $s+a_{1}<$ $s+m-1$ it follows that $P(n)<\left(\begin{array}{c}n+m-1 \\ n\end{array}\right)$ for all $n \geq s$. Define $f: \mathbb{N} \rightarrow \mathbb{N}$ by $f(n)=\left(\begin{array}{c}n+m-1 \\ n\end{array}\right)$ for $n<s$ and $f(n)=P(n)$ for $n \geq s$. Then $f(n+1) \leq$ $f(n)^{\langle n\rangle}$ for all $n$. Moreover, if $s>1$, then $f(1)=m$, and if $s=1$, then $f(1)=a_{1}+1<m$. By Theorem 3.1 we get $f=H_{I}$, and hence $P=P_{I}$, for some non-zero homogeneous ideal $I$ of $K[X]$.

The integers $a_{1}, \ldots, a_{s}$ describing a Hilbert polynomial $P$ as in the corollary are uniquely determined by $P$. For a homogeneous ideal $I$ of $K[X]$ let

$$
n_{0}(I):=\min \left\{n_{0} \in \mathbb{N}: H_{I}(n+1)=H_{I}(n)^{\langle n\rangle} \text { for all } n \geq n_{0}\right\} .
$$


If $E$ is a lex-segment, then $n_{0}\left(I_{E}\right)$ agrees with the largest degree of a minimal generator of $E$ (see [10, Corollary 4.2.9]). Note that given a Hilbert polynomial $P$ of a non-zero homogeneous ideal as in (3.1), the integer

$$
\varphi(P):=\min \left\{n_{0}(I): I \subseteq K[X] \text { homogeneous ideal with } P_{I}=P\right\}
$$

coincides with $s$ (by the proof of Corollary 3.2). We put $\varphi(I):=\varphi\left(P_{I}\right)$ for any non-zero homogeneous ideal $I$ of $K[X]$. We have $H_{I}(n)=P_{I}(n)$ for all $n \geq \varphi(I)$. We also note:

Corollary 3.3. Let $P(T) \in \mathbb{Q}[T]$ and $\operatorname{deg} P<m-1$. If $P$ is the Hilbert polynomial of some non-empty final segment of $\mathbb{N}^{n}$ (for some $n$ ), then $P$ is the Hilbert polynomial of some non-empty final segment of $\mathbb{N}^{m}$.

The ordered set of Hilbert polynomials. Let us write

$$
\mathcal{H}_{m}:=\left\{H_{E}: E \in \mathcal{F}\left(\mathbb{N}^{m}\right)\right\}
$$

for the set of Hilbert functions of final segments of $\mathbb{N}^{m}$, and put $\mathcal{H}:=$ $\bigcup_{m} \mathcal{H}_{m}$. We consider $\mathcal{H}$ as an ordered set via the product ordering:

$$
H_{E} \leq H_{F}: \Leftrightarrow H_{E}(s) \leq H_{F}(s) \text { for all } s .
$$

We have a strictly increasing surjection

$$
\left(\mathcal{F}\left(\mathbb{N}^{m}\right), \supseteq\right) \rightarrow \mathcal{H}_{m}: E \mapsto H_{E}
$$

Hence by Corollary 1.8:

COROllary 3.4. The ordered set $\mathcal{H}_{m}$ is Noetherian.

REMARK 3.5. In fact, the ordered set $\mathcal{H}$ is also Noetherian. This can be shown using Nash-Williams's theory of "better-quasi-orderings" (see [3]).

We write

$$
\mathcal{P}_{m}:=\left\{P_{E}: E \in \mathcal{F}\left(\mathbb{N}^{m}\right)\right\}
$$

for the set of Hilbert polynomials (of final segments of $\mathbb{N}^{m}$ ), and $\mathcal{P}:=$ $\bigcup_{m} \mathcal{P}_{m}$. We totally order $\mathcal{P}$ via the dominance ordering $\preceq$. Clearly $H_{E} \leq$ $H_{F} \Rightarrow P_{E} \preceq P_{F}$, so

$$
\mathcal{H}_{m} \rightarrow \mathcal{P}_{m}: H_{E} \mapsto P_{E}
$$

is an increasing surjection. A variant of the following fact has first been proved by Sit [41] using different methods:

COROLlary 3.6. The dominance ordering on the set $\mathcal{P}$ of Hilbert polynomials is a well-ordering.

Proof. By Corollary 3.4 and the preceding remarks, $\mathcal{P}_{m}$ is well-ordered for every $m$. Moreover, the leading coefficients of polynomials $P, Q \in \mathcal{P}$ are positive, so if $\operatorname{deg} P<\operatorname{deg} Q$, then $P \prec Q$. This implies that for every decreasing sequence $P_{0} \succeq P_{1} \succeq \cdots$ in $\mathcal{P}$ there exists some $m$ such that 
$P_{i} \in \mathcal{P}_{m}$ for all $i \gg 0$, and hence $P_{i}=P_{i+1}$ for all $i \gg 0$. This shows that $\mathcal{P}$ is well-ordered.

The following will be used in [4]:

Corollary 3.7. If $P, Q \in \mathcal{P}$ then $P+Q \in \mathcal{P}$ and $P \cdot Q \in \mathcal{P}$. That is, $\mathcal{P}$ is a subsemiring of the ring of all integer-valued polynomials.

Proof. By Corollary 3.3 we have $P=P_{I}$ and $Q=P_{J}$ for non-zero monomial ideals $I \subseteq \mathbb{Q}[X]$ and $J \subseteq \mathbb{Q}[Y]$, where $X=\left\{X_{1}, \ldots, X_{m}\right\}$ and $Y=$ $\left\{Y_{1}, \ldots, Y_{n}\right\}$ are disjoint sets of distinct indeterminates and $m=\operatorname{deg} P+2$, $n=\operatorname{deg} Q+2$. Consider the homomorphism of graded $\mathbb{Q}$-algebras

$$
\mathbb{Q}[X, Y] \rightarrow \mathbb{Q}[X] / I \oplus \mathbb{Q}[Y] / J
$$

defined by

$$
X_{i} \mapsto x_{i}=X_{i}+I \quad \text { and } \quad Y_{j} \mapsto y_{j}=Y_{j}+J \quad \text { for all } i, j .
$$

It is easy to see that its kernel $K_{0}$ is generated by

$$
I \cup J \cup\left\{X_{i} Y_{j}: 1 \leq i \leq m, 1 \leq j \neq n\right\}
$$

(hence is a monomial ideal of $\mathbb{Q}[X, Y]$ ), and

$$
\operatorname{dim}_{\mathbb{Q}}\left(\mathbb{Q}[X, Y] / K_{0}\right)_{s}=\operatorname{dim}_{\mathbb{Q}}(\mathbb{Q}[X] / I)_{s}+\operatorname{dim}_{\mathbb{Q}}(\mathbb{Q}[Y] / J)_{s}
$$

for all $s$ except possibly 0 . In particular, $P_{K_{0}}=P_{I}+P_{J} \in \mathcal{P}_{M}$, where $M=m+n$. As to $P \cdot Q$, it is well-known that $P \cdot Q=P_{S}$, where $S \subseteq$ $\mathbb{Q}\left[Z_{1}, \ldots, Z_{N}\right], N:=m \cdot n$, is the homogeneous ideal corresponding to the image of $V(I) \times V(J) \subseteq \mathbb{P}^{m-1} \times \mathbb{P}^{n-1}$ in $\mathbb{P}^{N-1}$ under the Segre embedding.

Remark 3.8. We write $\mathcal{P}_{m}^{*}:=\mathcal{P}_{m} \backslash\left\{P_{\emptyset}\right\}$, where $P_{\emptyset}=\left(\begin{array}{c}T+m-1 \\ m-1\end{array}\right)$. By the proof of the corollary $\mathcal{P}_{m}^{*}$ is closed under addition.

Hilbert-Samuel polynomials. We now associate another integervalued polynomial to a homogeneous ideal $I$ of $K[X]$. The function $h_{I}: \mathbb{N} \rightarrow$ $\mathbb{N}$ given by

$$
h_{I}(s)=\operatorname{dim}_{K}\left(R_{0} \oplus \cdots \oplus R_{s}\right) \quad \text { for } s \in \mathbb{N},
$$

where $R=K[X] / I$ as at the beginning of this section, is called the HilbertSamuel function of the ideal $I$. We put $h_{(1)}(s)=0$ for all $s$. If $E \in \mathcal{F}\left(\mathbb{N}^{m}\right)$ we put $h_{E}:=h_{I_{E}}$, where $I_{E}$ is the monomial ideal in $\mathbb{Q}[X]$ corresponding to $E$. With the notation $Z_{\leq s}:=\left\{z \in \mathbb{N}^{m}:|z| \leq s\right\}$ for $Z \subseteq \mathbb{N}^{m}$ and $s \in \mathbb{N}$ we then have $h_{E}(s)=\left|V_{E, \leq s}\right|$ for all $s$.

Lemma 3.9. Given a homogeneous ideal $I$ of $K[X]$ there exists an integer-valued polynomial $p_{I}$ of degree $\leq m$ such that $p_{I}(s)=h_{I}(s)$ for all $s \gg 0$ in $\mathbb{N}$.

Indeed, the function $h_{I}$ is nothing but the Hilbert function of the homogeneous ideal $I S$ of the polynomial ring $S:=K\left[X_{0}, X_{1}, \ldots, X_{m}\right]$. We 
call the polynomial $p_{I}$ the Hilbert-Samuel polynomial of the homogeneous ideal $I$. We put $p_{E}:=p_{I_{E}}$ for $E \in \mathcal{F}\left(\mathbb{N}^{m}\right)$.

Lemma 3.10. A polynomial $p(T) \in \mathbb{Q}[T]$ is the Hilbert-Samuel polynomial of a non-zero homogeneous ideal of the polynomial ring $K\left[X_{1}, \ldots, X_{m}\right]$ if and only if $p$ is the Hilbert polynomial of some non-zero homogeneous ideal of $K\left[X_{0}, X_{1}, \ldots, X_{m}\right]$.

Proof. The "only if" part follows from the preceding discussion. Conversely, suppose there exists a non-zero homogeneous ideal $I$ of $K\left[X_{0}, \ldots, X_{m}\right]$ such that $p=P_{I}$. We may assume that the maximal ideal $\left(X_{0}, \ldots, X_{m}\right)$ is not an associated prime of $I$. (Otherwise, $p=P_{I}=0$.) Then for a generic linear form $h \in K\left[X_{0}, \ldots, X_{m}\right]$, multiplication by $h$ on $R=K\left[X_{0}, \ldots, X_{m}\right] / I$ is injective (see [10, Proposition 1.5.12]). We have a short exact sequence of graded $K$-algebras and degree 0 maps:

$$
0 \rightarrow R(-1) \stackrel{h}{\rightarrow} R \rightarrow S \rightarrow 0,
$$

where $R(-1)=\bigoplus_{s \geq 0} R(-1)_{s}$ with $R(-1)_{0}=0, R(-1)_{s}=R_{s-1}$ for $s \geq 1$, and $S=K\left[X_{0}, \ldots, X_{m}\right] / J$ with $J=I+(h)$. Hence

$$
H_{I}(n)-H_{I}(n-1)=H_{J}(n) \quad \text { for } n \geq 1
$$

and so $H_{I}(n)=\sum_{i=0}^{n} H_{J}(i)$ for all $n$. Note that $H_{J}(1)=H_{I}(1)-1 \leq m$, so by Macaulay's Theorem there exists a non-zero homogeneous ideal $J^{\prime}$ of $K\left[X_{1}, \ldots, X_{m}\right]$ with $H_{J}=H_{J^{\prime}}$. Hence $H_{I}=h_{J}=h_{J^{\prime}}$ and thus $p=P_{I}$ $=p_{J^{\prime}}$

REMARK 3.11. We leave it to the reader to use the characterization of Hilbert polynomials established in Corollary 3.2 in conjunction with the previous lemma to formulate and prove an analogous characterization of Hilbert-Samuel polynomials. For the empty final segment $\emptyset \subseteq \mathbb{N}^{m}$ we have $p_{\emptyset}(T)=\left(\begin{array}{c}T+m \\ m\end{array}\right)$. If $p(T) \in \mathbb{Q}[T]$ has degree $m-1$ and is of the form $p=p_{F}$ for some non-empty final segment $F$ of $\mathbb{N}^{n}$ (for some $n$ ), then there exists a non-empty final segment $E$ of $\mathbb{N}^{m}$ with $p=p_{E}$.

Somewhat more generally, we can also define the Hilbert function $H_{E}$ and Hilbert polynomial $P_{E}$ of an $n$-tuple $E=\left(E_{1}, \ldots, E_{n}\right)$ of final segments of $\mathbb{N}^{m}$ by setting

$$
H_{E}=H_{E_{1}}+\cdots+H_{E_{n}}, \quad P_{E}=P_{E_{1}}+\cdots+P_{E_{n}} .
$$

Similarly we define the Hilbert-Samuel function $h_{E}$ and the Hilbert-Samuel polynomial $p_{E}$ of $E$. (We will use these constructions in [4].) Given $n$-tuples $E=\left(E_{1}, \ldots, E_{n}\right)$ and $F=\left(F_{1}, \ldots, F_{n}\right)$ of final segments of $\mathbb{N}^{m}$, we will write $E \supseteq F$ if $E_{i} \supseteq F_{i}$ for all $i=1, \ldots, n$; that is, $\supseteq$ denotes the product order on $\mathcal{F}\left(\mathbb{N}^{m}\right)^{n}$. The map that assigns to $E$ its Hilbert function has finite fibers. In fact: 
LEMMA 3.12. The maps

$$
E \mapsto H_{E}, \quad E \mapsto h_{E}, \quad E \mapsto p_{E}
$$

on $\mathcal{F}\left(\mathbb{N}^{m}\right)^{n}$ are strictly increasing and hence have finite fibers.

Proof. Let $E$ and $E^{\prime}$ be $n$-tuples of final segments of $\mathbb{N}^{m}$ with $E \supset E^{\prime}$. Then clearly $H_{E} \leq H_{E^{\prime}}$ (thus $h_{E} \leq h_{E^{\prime}}$ and $p_{E} \leq p_{E^{\prime}}$ ) and $H_{E} \neq H_{E^{\prime}}$. Say $H_{E}\left(s_{0}\right)<H_{E^{\prime}}\left(s_{0}\right)$ for some $s_{0}$; then $p_{E}(s)<p_{E^{\prime}}(s)$ for all $s \geq s_{0}$ sufficiently large. The rest now follows from Lemma 1.5(1) and the Noetherianity of $\mathcal{F}\left(\mathbb{N}^{m}\right)^{n}$.

It might be worth pointing out that although in general there are infinitely many final segments of $\mathbb{N}^{m}$ with a given Hilbert polynomial $P$ (for example, every non-empty final segment of $\mathbb{N}$ has Hilbert polynomial 0), it is not difficult to see that there always exists a smallest Hilbert function $H_{E}$ with $P_{E}=P$.

The ordered set of Hilbert-Samuel polynomials. We write

$$
\mathcal{S}_{m}:=\left\{p_{E}: E \in \mathcal{F}\left(\mathbb{N}^{m}\right)\right\}
$$

for the set of Hilbert-Samuel polynomials of final segments of $\mathbb{N}^{m}$, and $\mathcal{S}:=\bigcup_{m} \mathcal{S}_{m}$. We also let $\mathcal{S}_{m}^{*}:=\mathcal{S}_{m} \backslash\left\{p_{\emptyset}\right\}$ with $p_{\emptyset}=\left(\begin{array}{c}T+m \\ m\end{array}\right)$. By Lemma 3.10, we have $\mathcal{S}_{m}^{*}=\mathcal{P}_{m+1}^{*}$ for all $m$. By Corollaries 3.6 and 3.7:

COROLlary 3.13. The set $\mathcal{S}$ is a well-ordered subsemiring of the ordered ring of integer-valued polynomials.

Corollary 3.2 of Macaulay's Theorem makes it possible to describe the unique isomorphism between $\mathcal{S}_{m}$ and its order type o $\left(\mathcal{S}_{m}\right)$ in a rather explicit way:

Definition 3.14. Every Hilbert-Samuel polynomial $p(T)$ of a nonempty final segment of $\mathbb{N}^{m}$ can be written uniquely in the form

$$
p(T)=\left(\begin{array}{c}
T+a_{1} \\
a_{1}
\end{array}\right)+\left(\begin{array}{c}
T+a_{2}-1 \\
a_{2}
\end{array}\right)+\cdots+\left(\begin{array}{c}
T+a_{s}-(s-1) \\
a_{s}
\end{array}\right)
$$

for certain integers $m>a_{1} \geq a_{2} \geq \cdots \geq a_{s} \geq 0$ and $s \geq 0$. We put

$$
\mathbf{c}_{p}:=\left(c_{m-1}, c_{m-2}, \ldots, c_{0}\right) \in \mathbb{N}^{m}
$$

where $c_{i}$ denotes the number of occurrences of $i \in\{0, \ldots, m-1\}$ among the coefficients $a_{1}, a_{2}, \ldots, a_{s}$. Note that $\left|\mathbf{c}_{p}\right|=s$. We define an ordinal

$$
\psi_{p}:=\omega^{m-1} c_{m-1}+\omega^{m-2} c_{m-2}+\cdots+c_{0} .
$$

For the Hilbert-Samuel polynomial $p(T)=\left(\begin{array}{c}T+m \\ m\end{array}\right)$ of the empty subset of $\mathbb{N}^{m}$ we set $\psi_{p}:=\omega^{m}$.

The following observation is now easy. (This gives another proof of the well-orderedness of $\mathcal{S}_{m}$.) 
Corollary 3.15. The map

$$
p \mapsto \psi_{p}: \mathcal{S}_{m} \rightarrow \omega^{m}+1,
$$

is an isomorphism of ordered sets.

In [4] we will use the previous corollary to define a new model-theoretic rank for definable sets in differentially closed fields of characteristic zero (via their Kolchin polynomials, see [22] or [23]).

Computation of $\psi_{p}$. Here is how the $\mathbf{c}_{p}$ can be computed recursively, following [23]. (In [23], $c_{0}, \ldots, c_{m-1}$ are called the minimizing coefficients of $p$.) Let

$$
p(T)=b_{d}\left(\begin{array}{c}
T+d \\
d
\end{array}\right)+b_{d-1}\left(\begin{array}{c}
T+d-1 \\
d-1
\end{array}\right)+\cdots+b_{0}\left(\begin{array}{c}
T+0 \\
0
\end{array}\right)
$$

with $b_{0}, \ldots, b_{d} \in \mathbb{Z}, b_{d} \neq 0$, be an integer-valued polynomial of degree $d$. We define a sequence $\widetilde{\mathbf{c}}_{p} \in \mathbb{Z}^{m}$, where $m=d+1$, by induction on $d$ as follows: If $p=0$ or $d=0$, so $p(T)=b_{0}$ is constant, we put $\widetilde{\mathbf{c}}_{p}:=\left(b_{0}\right)$. If $d>1$, we consider the integer-valued polynomial

$$
q(T):=p\left(T+b_{d}\right)-\left(\begin{array}{c}
T+d+1+b_{d} \\
d+1
\end{array}\right)+\left(\begin{array}{c}
T+d+1 \\
d+1
\end{array}\right) .
$$

Note that $e:=\operatorname{deg} q<d$, so $\widetilde{\mathbf{c}}_{q}=\left(\widetilde{c}_{q, e}, \ldots, \widetilde{c}_{q, 0}\right) \in \mathbb{Z}^{e+1}$ has been defined already. We let $\widetilde{\mathbf{c}}_{p}:=\left(b_{d}, 0, \ldots, 0, \widetilde{c}_{q, e}, \ldots, \widetilde{c}_{q, 0}\right) \in \mathbb{Z}^{m}$.

LEMma 3.16. $p(T) \in \mathcal{S}_{m}^{*}$ if and only if $\widetilde{\mathbf{c}}_{p} \geq 0$, and in this case $\widetilde{\mathbf{c}}_{p}=\mathbf{c}_{p}$.

Proof. We proceed by induction on $d$. The case $d=0$ is trivial. Suppose $d>0$, and assume first that $p(T) \in \mathcal{S}_{m}^{*}$, say $p(T)=p_{I}(T)$ for some non-zero monomial ideal $I$ of $R=\mathbb{Q}\left[X_{1}, \ldots, X_{m}\right]$. Since $p(s)>0$ for $s \gg 0$, we clearly have $b_{d}>0$. For $i=1, \ldots, m$ we let $\nu_{i} \in \mathbb{N}$ be the smallest natural number such that $X_{i}^{\nu_{i}} X^{\mu} \in I$ for some $\mu \in \mathbb{N}^{m}$ with $\mu_{i}=0$. Multiplication by $X^{\nu}$, where $\nu=\left(\nu_{1}, \ldots, \nu_{m}\right)$, induces a short exact sequence

$$
0 \rightarrow\left(R /\left(I: X^{\nu}\right)\right)(-|\nu|) \stackrel{X^{\nu}}{\longrightarrow} R / I \rightarrow R /\left(X^{\nu}\right) \rightarrow 0 .
$$

Hence for all $s$,

$$
H_{\left(I: X^{\nu}\right)}(s)=H_{I}(s+|\nu|)-H_{\left(X^{\nu}\right)}(s+|\nu|) .
$$

Using the short exact sequences

$$
0 \rightarrow\left(R /\left(I: X_{i}^{\nu_{i}}\right)\right)\left(-\left|\nu_{i}\right|\right) \stackrel{X_{i}^{\nu_{i}}}{\longrightarrow} R / I \rightarrow R /\left(X_{i}^{\nu_{i}}\right) \rightarrow 0
$$

for $i=1, \ldots, m$ it is easy to see that $|\nu|=b_{d}$. It follows that

$$
h_{\left(I: X^{\nu}\right)}(s)=p_{I}\left(s+b_{d}\right)-\left(\begin{array}{c}
s+d+1+b_{d} \\
d+1
\end{array}\right)+\left(\begin{array}{c}
s+d+1 \\
d+1
\end{array}\right)
$$


for all $s \gg 0$, and therefore $q=p_{\left(I: X^{\nu}\right)}$. By induction we get $\widetilde{\mathbf{c}}_{q} \geq 0$ and thus $\widetilde{\mathbf{c}}_{p} \geq 0$. Conversely, suppose that $\widetilde{\mathbf{c}}_{p} \geq 0$. By induction we may write $q=p_{J}$ for some monomial ideal $J$ of $R^{\prime}=K\left[X_{1}, \ldots, X_{m-1}\right]$. We put $I=$ $\left(X_{m}^{b_{d}+1}, X_{m}^{b_{d} J}\right)$, a monomial ideal of $R=K\left[X_{1}, \ldots, X_{m}\right]$. Then, using the short exact sequence

$$
0 \rightarrow\left(R /\left(X_{m}, J\right)\right)\left(-b_{d}\right) \stackrel{X_{m}^{b_{d}}}{\longrightarrow} R / I \rightarrow R /\left(X_{m}^{b_{d}}\right) \rightarrow 0
$$

and the fact that $R /\left(X_{m}, J\right) \cong R^{\prime} / J$ we obtain $p=p_{I}$ as required. The identity $\widetilde{\mathbf{c}}_{p}=\mathbf{c}_{p}$ follows from Corollary 3.15, Lemma 2.3(1) and the observation that $\left(\mathcal{S}_{m}^{*}, \preceq\right) \rightarrow\left(\mathbb{N}^{m}, \leq_{\text {lex }}\right): p \mapsto \widetilde{\mathbf{c}}_{p}$ is strictly increasing and surjective.

EXAMPLE 3.17. Let $p(T)=a(T+1)+b \in \mathbb{Z}[T]$ with $a, b \in \mathbb{Z}, a \neq 0$. Then $p(T)$ is the Hilbert-Samuel polynomial of a non-empty final segment of $\mathbb{N}^{2}$ if and only if $a>0$ and $b+\left(\begin{array}{c}a \\ 2\end{array}\right) \geq 0$. In this case, the sequence $\mathbf{c}_{p}$ is given by $\left(a, b+\left(\begin{array}{l}a \\ 2\end{array}\right)\right)$, so $\psi_{p}=\omega a+b+\left(\begin{array}{l}a \\ 2\end{array}\right)$. (If we write $p(T)=d T+1-g$ this yields the well-known inequality $g \leq\left(\begin{array}{c}d-1 \\ 2\end{array}\right)$ relating degree and genus of a projective curve.)

Application: length of increasing chains of ideals. The results of this section, in particular Corollary 3.15, can be used to study increasing chains of ideals in polynomial rings. We give two applications. First let us prove the theorem stated in the introduction. We denote the set of homogeneous ideals of $K[X]=K\left[X_{1}, \ldots, X_{m}\right]$, ordered by reverse inclusion, by $\mathcal{I}_{m}$. Since $K[X]$ is Noetherian, $\mathcal{I}_{m}$ is well-founded. We write $p: \mathcal{I}_{m} \rightarrow \mathcal{S}_{m}$ for the map $I \mapsto p_{I}$.

Lemma 3.18. $\operatorname{ht}\left(\mathcal{I}_{m}\right)=\operatorname{ht}\left(\mathcal{F}\left(\mathbb{N}^{m}\right)\right)=\omega^{m}+1$.

Proof. The first equality holds since there exists a strictly increasing surjection $\mathcal{I}_{m} \rightarrow \mathcal{F}\left(\mathbb{N}^{m}\right)$. This is a well-known consequence of the division algorithm in $K[X]$ (see, e.g., [15, Chapter 15]): Choose a term ordering $\leq$ on $X^{\diamond}$; given a non-zero polynomial $f \in K[X] \operatorname{let} \operatorname{lm}(f)$ be the leading monomial of $f$, that is, the largest monomial in the ordering $\leq$ which occurs in $f$ with a non-zero coefficient. Given an ideal $I$ of $K[X]$ we denote by $\operatorname{lm}(I)$ the monomial ideal generated by the $\operatorname{lm}(f)$, where $0 \neq f \in I$. Now suppose that $I \supset J$ are ideals in $K[X]$. Then $\operatorname{lm}(I) \supset \operatorname{lm}(J)$ : Choose $f \in I \backslash J$ such that $\operatorname{lm}(f)$ is minimal in the ordering $\leq$; we claim that $\operatorname{lm}(f) \in \operatorname{lm}(I) \backslash \operatorname{lm}(J)$. Otherwise $\operatorname{lm}(f)=\operatorname{lm}(g)$ for some $0 \neq g \in J$, and we can write $f=q g+r$ for some $q, r \in K[X], r \neq 0$, with $\operatorname{lm}(r)<\operatorname{lm}(f)$. Since $r \in I \backslash J$, this is a contradiction. Hence the map which associates to a homogeneous ideal $I$ the monomial ideal $\operatorname{lm}(I)$ is strictly increasing. The second equality follows from Proposition 2.11.

We now show: 
THEOREM 3.19. For every strictly increasing surjection $\varphi: \mathcal{I}_{m} \rightarrow S$, where $S$ is an ordered set, there exists a non-decreasing map $\psi: \mathcal{S}_{m} \rightarrow S$ such that $\psi \circ p \leq \varphi$.

Proof. The map $I \mapsto \psi_{p_{I}}: \mathcal{I}_{m} \rightarrow \omega^{m}+1$ is strictly increasing and surjective. Hence $\psi_{p_{I}}=\operatorname{ht}_{\mathcal{I}_{m}}(I)$ for all $I \in \mathcal{I}_{m}$, by the last lemma. In fact, $\mathrm{ht}_{\mathcal{I}_{m}}=\mathrm{ht}_{\mathcal{S}_{m}} \circ p$. The claim now follows from Lemma 2.3(2).

(By the second part of Lemma 2.3, the function $p: \mathcal{I}_{m} \rightarrow \mathcal{S}_{m}$ is characterized up to isomorphism by the property expressed in Theorem 3.19, in the category of strictly increasing surjections $\mathcal{I}_{m} \rightarrow S$, where $S$ is a totally ordered set.)

REMARK 3.20. Let $\mathcal{G}_{m}$ denote the set of isomorphism classes of finitely generated graded $R$-modules, where $R=K[X]$. We define a binary relation $\leq$ on $\mathcal{G}_{m}$ by $M \leq N \Leftrightarrow$ there exists a surjective homomorphism of graded $R$-modules $N \rightarrow M$. Since every surjective endomorphism of a finitely generated $R$-module is an isomorphism (see [15]), it follows that $\leq$ is an ordering on $\mathcal{G}_{m}$. By Noetherianity of $R$, $\leq$ is well-founded. We ask: Does Theorem 3.19 remain true when $\mathcal{I}_{m}$ is replaced by $\mathcal{G}_{m}$ and $p$ by the map assigning to $M \in \mathcal{G}_{m}$ its Hilbert-Samuel polynomial?

By the theorem above, every strictly increasing chain of non-zero homogeneous ideals in $K[X]$ gives rise to a strictly decreasing sequence in the lexicographically ordered set $\omega^{m}$. What can be said about the length of such sequences? For this, let us fix an increasing function $f: \mathbb{N} \rightarrow \mathbb{N}$, and consider finite sequences

$$
\nu_{0}>_{\text {lex }} \nu_{1}>_{\text {lex }} \cdots>_{\text {lex }} \nu_{\ell-1}
$$

of $m$-tuples $\nu_{i} \in \mathbb{N}^{m}$, strictly decreasing with respect to the lexicographic ordering on $\mathbb{N}^{m}$, with the property that $\left|\nu_{i}\right| \leq f(i)$ for all $i$. For the purpose of this section, let us call such a sequence an $f$-bounded sequence in $\mathbb{N}^{m}$. By König's Lemma (e.g., [20, p. 20]) applied to the tree whose nodes are the $f$-bounded sequences it follows that there exists an $f$-bounded sequence with maximal length $\ell=\ell(m, f)$. It is not difficult to compute an explicit formula for $\ell(m, f)$ :

Lemma 3.21. We have $\ell(1, f)=f(0)+1$ and

$$
\ell(m, f)=1+\ell\left(m-1, f_{1}\right)+\cdots+\ell\left(m-1, f_{f(0)}\right) \quad \text { for } m>1
$$

with $f_{i}: \mathbb{N} \rightarrow \mathbb{N}$ defined by

$$
f_{i}(j)=f\left(j+1+\ell\left(m-1, f_{1}\right)+\cdots+\ell\left(m-1, f_{i-1}\right)\right)-f(0)+i
$$

for $i, j \in \mathbb{N}, i \geq 1$. 
Proof. By induction on $m$. The case $m=1$ is trivial. Suppose that $m>1$, and let

$$
\nu_{0}>_{\text {lex }} \nu_{1}>_{\text {lex }} \cdots>_{\text {lex }} \nu_{\ell-1}
$$

be an $f$-bounded sequence in $\mathbb{N}^{m}$ of maximal length $\ell=\ell(m, f)$. We must have $\nu_{0}=(f(0), 0, \ldots, 0)$; otherwise (since $f$ is increasing)

$$
(f(0), 0, \ldots, 0)>_{\text {lex }} \nu_{0}>_{\text {lex }} \nu_{1}>_{\text {lex }} \cdots>_{\text {lex }} \nu_{\ell-1}
$$

would be a longer $f$-bounded sequence. For a similar reason, $\nu_{1}$ must have the form $\nu_{1}=(f(0)-1) \mu_{0}$ for some $\mu_{0} \in \mathbb{N}^{m-1}$. It follows that

$$
\nu_{1}=(f(0)-1) \mu_{0}, \ldots, \nu_{\ell_{1}}=(f(0)-1) \mu_{\ell_{1}-1}
$$

for some $f_{1}$-bounded sequence $\mu_{0}>_{\text {lex }} \mu_{1}>_{\text {lex }} \cdots>_{\text {lex }} \mu_{\ell_{1}-1}$ in $\mathbb{N}^{m-1}$ of maximal length $\ell_{1}=\ell\left(m-1, f_{1}\right)$. The next terms in the sequence must then have the form $\nu_{\ell_{1}+i}=(f(0)-2) \lambda_{i-1}$ for some $f_{2}$-bounded sequence $\lambda_{0}>_{\text {lex }} \lambda_{1}>_{\text {lex }} \cdots>_{\text {lex }} \lambda_{\ell_{2}-1}$ in $\mathbb{N}^{m-1}$ of maximal length $\ell_{2}=\ell\left(m-1, f_{2}\right)$, and so on. This leads to the formula $(3.2)$ for $\ell(m, f)$.

We can use this to show the following statement about uniform bounds for the length of ascending chains of homogeneous ideals. Recall that for any homogeneous ideal $I$ of $K[X]=K\left[X_{1}, \ldots, X_{m}\right]$, we denote by $\varphi(I)$ the smallest natural number $n_{0}$ such that for any homogeneous ideal $J$ of $K[X]$ with Hilbert polynomial $P_{J}=P_{I}$, we have $H_{J}(n+1)=H_{J}(n)^{\langle n\rangle}$ for all $n \geq n_{0}$. (Cf. remarks following Corollary 3.2.)

Proposition 3.22. Let $f: \mathbb{N} \rightarrow \mathbb{N}$ be any function and $m \geq 1$. There exists a natural number $t_{m}(f)$ depending only on $m$ and $f$, and primitive recursive in $f$, such that for any field $K$ and any strictly increasing chain

$$
I_{0} \subset I_{1} \subset \cdots \subset I_{t-1}
$$

of non-zero homogeneous ideals in $K\left[X_{1}, \ldots, X_{m}\right]$ such that $\varphi\left(I_{i}\right) \leq f(i)$ for all $i$, we have $t \leq t_{m}(f)$.

Here as usual, a function $F: \mathbb{N}^{r} \rightarrow \mathbb{N}$ (for $r \in \mathbb{N}$ ) is called primitive recursive in a given collection $F_{1}, \ldots, F_{k}$ of functions $F_{i}: \mathbb{N}^{r_{i}} \rightarrow \mathbb{N}$ if it can be obtained from $F_{1}, \ldots, F_{k}$ as well as the constant function 0 , the successor function $x \mapsto x+1$, coordinate permutations $\left(x_{1}, \ldots, x_{n}\right) \mapsto\left(x_{\sigma(1)}, \ldots, x_{\sigma(n)}\right)$, and the projections $\left(x_{1}, \ldots, x_{n+1}\right) \mapsto\left(x_{1}, \ldots, x_{n}\right): \mathbb{N}^{n+1} \rightarrow \mathbb{N}^{n}$, by finitely many applications of the following rules (substitution and induction, respectively):

(1) if $F: \mathbb{N}^{r} \rightarrow \mathbb{N}$ and $G_{1}, \ldots, G_{r}: \mathbb{N}^{s} \rightarrow \mathbb{N}$ are primitive recursive in $F_{1}, \ldots, F_{k}$, then so is $H=F\left(G_{1}, \ldots, G_{r}\right): \mathbb{N}^{s} \rightarrow \mathbb{N}$

(2) if $F: \mathbb{N}^{r} \rightarrow \mathbb{N}$ and $G: \mathbb{N}^{r+2} \rightarrow \mathbb{N}$ are primitive recursive in $F_{1}, \ldots, F_{k}$, 
then so is the function $H: \mathbb{N}^{r+1} \rightarrow \mathbb{N}$ defined by

$$
H(x, y)= \begin{cases}F(x) & \text { if } y=0 \\ G(x, y-1, H(x, y-1)) & \text { if } y>0\end{cases}
$$

for $x \in \mathbb{N}^{r}$ and $y \in \mathbb{N}$.

If $k=0$, we obtain the plain primitive recursive functions. Standard numbertheoretic functions like addition $(x, y) \mapsto x+y$, multiplication $(x, y) \mapsto x \cdot y$ or exponentiation $(x, y) \mapsto x^{y}$ are primitive recursive. The class of primitive recursive functions forms a proper subclass of all recursive, or computable, functions. A prominent example of a recursive but not primitive recursive function is the Ackermann function. (See, e.g., [30], for the definition of the Ackermann function.)

Before we prove Proposition 3.22 we show the following lemma. Given $Q(T) \in \mathbb{Q}[T]$ we put $\Delta Q(T)=Q(T)-Q(T-1) \in \mathbb{Q}[T]$. Note that if $\Delta Q=P$ and $Q(0)=P(0)$, then $Q(n)=\sum_{i=0}^{n} P(i)$ for all $n \in \mathbb{N}$.

\section{LEMMA 3.23. Let}

$$
P(T)=\left(\begin{array}{c}
T+a_{1} \\
a_{1}
\end{array}\right)+\left(\begin{array}{c}
T+a_{2}-1 \\
a_{2}
\end{array}\right)+\cdots+\left(\begin{array}{c}
T+a_{s}-(s-1) \\
a_{s}
\end{array}\right)
$$

with integers $a_{1} \geq a_{2} \geq \cdots \geq a_{s} \geq 0$ and $s \geq 1$. Then $Q(T)=$

$$
\left(\begin{array}{c}
T+\left(a_{1}+1\right) \\
a_{1}+1
\end{array}\right)+\left(\begin{array}{c}
T+\left(a_{2}+1\right)-1 \\
a_{2}+1
\end{array}\right)+\cdots+\left(\begin{array}{c}
T+\left(a_{s}+1\right)-(s-1) \\
a_{s}+1
\end{array}\right)
$$

is the unique polynomial in $\mathbb{Q}[T]$ such that $Q(0)=P(0)$ and $\Delta Q=P$.

Proof. Clearly $Q(T)$ satisfies $Q(0)=1=P(0)$. The well-known identity $\left(\begin{array}{l}a \\ b\end{array}\right)-\left(\begin{array}{c}a-1 \\ b\end{array}\right)=\left(\begin{array}{c}a-1 \\ b-1\end{array}\right)$ for $a \geq b \geq 0$ implies $\Delta Q=P$ as required.

Proof of Proposition 3.22. First replacing $f$ by the function $g: \mathbb{N} \rightarrow \mathbb{N}$ defined by by $i \mapsto \max \{f(0), \ldots, f(i)\}$, if necessary, we may assume that $f$ is increasing. If $I$ is a non-zero homogeneous ideal in $K[X]$ with HilbertSamuel polynomial $p=p_{I}$ and $s=\varphi(I)$, then for $n \geq s$ we have

$$
p(n)=\sum_{i=0}^{n} H_{I}(i)=Q(n)+k,
$$

where $Q(n)=\sum_{i=0}^{n} P_{I}(i)$ and $k=\sum_{i=0}^{s-1}\left(H_{I}(i)-P_{I}(i)\right)$. By the previous lemma it follows that $\left|\mathbf{c}_{p}\right|=s+k$, and since $j \leq\left(\begin{array}{c}s-1+m \\ m\end{array}\right)$, we have $\left|\mathbf{c}_{p}\right| \leq$ $s+\left(\begin{array}{c}s-1+m \\ m\end{array}\right)=: h_{m}(s)$. Hence the function $t_{m}(f):=\ell\left(m, h_{m} \circ f\right)$, with $\ell$ as defined in Lemma 3.21, bounds the length of every strictly increasing chain of ideals as in Proposition 3.22. It is a tedious but straightforward exercise, left to the reader, to verify that $t_{m}(f)$ is primitive recursive in $f$, for given $m$. 
The proposition above yields the following theorem of Moreno Socías [30]:

Corollary 3.24. Let $f: \mathbb{N} \rightarrow \mathbb{N}$ be any function and $m \geq 1$. There exists a natural number $t_{m}(f)$ which is primitive recursive in $f$ such that for any field $K$ and any strictly increasing chain

$$
I_{0} \subset I_{1} \subset \cdots \subset I_{t-1}
$$

of ideals in $K[X]=K\left[X_{1}, \ldots, X_{m}\right]$ such that $I_{i}$ is generated by polynomials of degree at most $f(i)$, for every $i$, we have $t \leq t_{m}(f)$.

Proof. First we show that we may restrict ourselves to chains (3.3) where each ideal $I_{i}$ is monomial. To see this choose a term ordering $\leq$ on $X^{\diamond}$ which is degree-compatible. Then, as in the proof of the first equality in Lemma 3.18, one shows that if $I \subset J$ are ideals and $J$ is generated by polynomials of degree $\leq d$, then there exists a monomial $X^{\nu} \in \operatorname{lm}(J) \backslash \operatorname{lm}(I)$ of degree $|\nu| \leq d$. As in [30, Section 4] one further reduces to the case where every monomial ideal $I_{i}$ is of the form $I_{i}=I_{E_{i}}$ for a lex-segment $E_{i} \subseteq \mathbb{N}^{m}$. By the remarks following Corollary 3.2 we then have $\varphi\left(I_{i}\right) \leq f(i)$ for all $i$. Hence $t_{m}(f)$ as defined in the previous proposition works.

In [30, Corollary 7.5] it is also shown that $t_{m}(f)$ is not primitive recursive in $m$, even for an affine function $f(i)=p+i q(p, q \in \mathbb{N})$. In fact, $m \mapsto t_{m}(f)$ grows like the Ackermann function, and hence extremely rapidly.

Moreno Socías's result 3.24 may be interpreted as a quantitative variant of Dickson's Lemma (and thus, of the Hilbert Basis Theorem). We finish this section with outlining the proof of a similar finitary formulation of Maclagan's principle. This fact can be seen to provide primitive recursive complexity estimates for algorithms whose termination has been shown using the Noetherianity of $\mathcal{F}\left(\mathbb{N}^{m}\right)$. The proof is based on ideas of Harvey Friedman [17]. It also gives a different argument for Proposition 3.24 in the case where $f$ is affine, by reducing to the case of ascending chains of monomial ideals, as in the argument at the beginning of the proof of 3.24.

Proposition 3.25. Let $p, q \in \mathbb{N}, m \geq 1$. There exists a natural number $r_{m}(p, q)$, which is primitive recursive in $p$ and $q$, such that for any bad sequence

$$
F_{0}, F_{1}, \ldots, F_{r-1}
$$

of final segments of $\mathbb{N}^{m}$, with $F_{i}$ generated by elements of degree at most $p+i q$, we have $r \leq r_{m}(p, q)$.

Sketch of the proof. We fix $m \geq 1$. Let $T$ be the first-order theory of the structure $\mathbf{N}=(\mathbb{N}, \leq)$ in the language $\mathcal{L}_{0}$ consisting of the binary relation symbol $\leq$ and a constant symbol for every element of $\mathbb{N}$. Every model of $T$ is an ordered set containing an isomorphic copy of $\mathbf{N}$ as an initial segment, 
which we identify with $\mathbf{N}$. For $r \in \mathbb{N}$ we let $\mathcal{L}_{r}$ be the language $\mathcal{L}$ augmented by $m$-ary predicate symbols $F_{0}, \ldots, F_{r-1}$. For $p, q, r \in \mathbb{N}$ let $T_{p, q, r}$ be the union of the $\mathcal{L}_{r}$-theory $T$ together with sentences that express that $F_{0}, \ldots, F_{r-1}$ form a bad sequence of final segments, and each $F_{i}$ is generated by elements of $\mathbb{N}^{m}$ of degree $\leq p+i q$. Given $p, q \in \mathbb{N}$, the set $T_{p, q}=\bigcup_{r} T_{p, q, r}$ of sentences in the language $\mathcal{L}=\bigcup_{r} \mathcal{L}_{r}$ is inconsistent, by Noetherianity of $\mathcal{F}\left(\mathbb{N}^{m}\right)$. The Completeness Theorem of first-order logic implies that for some $r, T_{p, q, r}$ is inconsistent. Clearly any such $r$ bounds the length of a bad sequence in $\mathcal{F}\left(\mathbb{N}^{m}\right)$ with the $i$ th element in the sequence generated in degrees $\leq p+i q$.

In order to show that $r$ can be found primitive recursively in $p, q$, we use some facts about the so-called second principal system of reverse mathematics $\mathrm{WKL}_{0}$ (see [40]): First, the proof of the Completeness Theorem for countable languages can be carried out in $\mathrm{WKL}_{0}$ [40, Section IV.3]. It is a routine exercise to verify that the Noetherianity of $\mathcal{F}\left(\mathbb{N}^{m}\right)$ is also provable in $\mathrm{WKL}_{0}$. (For example, the proof given in the next section can be easily formalized.) The inconsistency of $T_{p, q, r}$ can be expressed by an existential formula $\varphi(p, q, r)$ in the language of arithmetic, and the $\forall \exists$ sentence $\forall p \forall q \exists r \varphi(p, q, r)$ is provable in $\mathrm{WKL}_{0}$. By a theorem of Friedman and Harrington [40, IX.3] there exists a primitive recursive function $\mathbb{N}^{2} \rightarrow \mathbb{N}$ : $(p, q) \mapsto r_{m}(p, q)$ such that $\mathrm{WKL}_{0} \vdash \forall p \forall q \varphi\left(p, q, r_{m}(p, q)\right)$. Our claim follows.

REMARK 3.26. The precise form of bounding function $i \mapsto p+i q$ used in Proposition 3.25 is not essential: Let $g: \mathbb{N}^{k+1} \rightarrow \mathbb{N}$ be a primitive recursive function, $k \in \mathbb{N}$. Then there exists a primitive recursive function $r_{m, g}: \mathbb{N}^{k} \rightarrow \mathbb{N}$ such that that for any $p_{1}, \ldots, p_{k} \in \mathbb{N}$ and any bad sequence $F_{0}, F_{1}, \ldots, F_{r-1}$ of final segments of $\mathbb{N}^{m}$, with $F_{i}$ generated by elements of degree at most $g\left(p_{1}, \ldots, p_{k}, i\right)$, we have $r \leq r_{m, g}\left(p_{1}, \ldots, p_{k}\right)$.

It is well-known that given an ideal $I=\left(f_{1}, \ldots, f_{n}\right)$ in a polynomial ring $K[X]=K\left[X_{1}, \ldots, X_{m}\right]$ over a field $K$, with $\operatorname{deg} f_{i} \leq d$ for all $d$, the ideal $\operatorname{lm}(I)$ of leading monomials of elements of $I$, with respect to a degreecompatible term ordering, can be generated by monomials whose degree is bounded by $d^{2^{m}}$ (see, e.g., [14]). Since this bound is primitive recursive in $d$, Remark 3.26 (applied to $g: \mathbb{N}^{3} \rightarrow \mathbb{N}$ given by $g(p, q, i)=(p+i q)^{2^{m}}$ ) implies:

Corollary 3.27. Let $p, q \in \mathbb{N}, m \geq 1$. There exists a natural number $s_{m}(p, q)$, which is primitive recursive in $p$ and $q$, such that for any field $K$ and any sequence

$$
I_{0}, I_{1}, \ldots, I_{s-1}
$$

of ideals of $K\left[X_{1}, \ldots, X_{m}\right]$ with $s \geq s_{m}(p, q)$ and with each $I_{i}$ generated by elements of degree at most $p+i q$, there exists $0 \leq i<j<s$ such that $\operatorname{lm}\left(I_{i}\right) \supseteq \operatorname{lm}\left(I_{j}\right)$ (and hence in particular $H_{I_{i}} \leq H_{I_{j}}$ ). 


\section{TOTAL ORDERINGS OF MONOMIAL IDEALS}

In this section, we study the ordered set $\mathcal{F}\left(\mathbb{N}^{m}\right)$ of final segments of $\mathbb{N}^{m}$, with the ordering given by the superset relation (see Example 1.2). We give an upper bound on o $\left(\mathcal{F}\left(\mathbb{N}^{m}\right)\right)$ and we explicitly describe several ways of extending the ordering on $\mathcal{F}\left(\mathbb{N}^{m}\right)$ to a well-ordering. Finally, we compute the order type of one particularly useful ordering, called the Kleene-Brouwer ordering of $\mathcal{F}\left(\mathbb{N}^{m}\right)$.

Bounding the type of $\mathcal{F}\left(\mathbb{N}^{m}\right)$. Our computation of an upper bound for the type of $\mathcal{F}\left(\mathbb{N}^{m}\right)$ is based on the following idea (which, incidentally, gives yet another proof of the Noetherianity of $\mathcal{F}\left(\mathbb{N}^{m}\right)$ ). Recall that an ideal in a commutative ring is called irreducible if it cannot be written as the intersection of two strictly larger ideals. (For example, prime ideals are irreducible.) By Noetherianity of $K[X]$, every ideal $I$ of $K[X]$ (where $K$ is a field) can be written as an intersection of irreducible ideals. Such a representation $I=J_{1} \cap \cdots \cap J_{r}(r \in \mathbb{N}, r>0)$ of $I$ as an intersection of irreducible ideals $J_{1}, \ldots, J_{r}$ is not, however, necessarily unique, even if we require it to be irredundant, that is, $J_{i} \nsubseteq J_{j}$ for $i \neq j$. However, an irredundant decomposition $I=J_{1} \cap \cdots \cap J_{r}$ of a monomial ideal $I$ is unique, and in this case the irreducible components $J_{i}$ are monomial ideals as well. It is easy to see that every irreducible monomial ideal is of the form $\mathfrak{m}^{\nu}:=$ $\left(X_{i}^{\nu_{i}}: \nu_{i}>0\right)$ for some $\nu=\left(\nu_{1}, \ldots, \nu_{m}\right) \in \mathbb{N}^{m}$. Note that

$$
\begin{aligned}
& {\left[\nu^{(1)}, \ldots, \nu^{(r)}\right] \leq^{\diamond}\left[\mu^{(1)}, \ldots, \mu^{(s)}\right] \text { in }\left(\mathbb{N}^{m}\right)^{\diamond} } \\
& \Rightarrow \mathfrak{m}^{\nu^{(1)}} \cap \cdots \cap \mathfrak{m}^{\nu^{(r)}} \supseteq \mathfrak{m}^{\mu^{(1)}} \cap \cdots \cap \mathfrak{m}^{\mu^{(s)}}
\end{aligned}
$$

for all $\nu^{(i)}, \mu^{(j)} \in \mathbb{N}^{m}$ such that $\operatorname{supp}\left(\nu^{(i)}\right) \supseteq \operatorname{supp}\left(\mu^{(j)}\right)$ for all $i, j$. Here $\operatorname{supp} \nu=\left\{i: \nu_{i}>0\right\}$ denotes the support of $\nu=\left(\nu_{1}, \ldots, \nu_{m}\right) \in \mathbb{N}^{m}$. (See Section 1 for the definition of $\leq^{\diamond}$.) Given a vector $\nu=\left(\nu_{1}, \ldots, \nu_{m}\right) \in \mathbb{N}^{m}$ let us write $\langle\nu\rangle:=\left(\nu_{i_{1}}, \ldots, \nu_{i_{k}}\right) \in \mathbb{N}^{k}$, where $1 \leq i_{1}<\cdots<i_{k} \leq m$ are the elements of the support of $\nu$ listed in increasing order. Given a final segment $E \in \mathcal{F}\left(\mathbb{N}^{m}\right)$ and $\sigma \subseteq\{1, \ldots, m\}$ we denote by $\varphi(E, \sigma)$ the commutative word $\left[\left\langle\nu^{(1)}\right\rangle, \ldots,\left\langle\nu^{(r)}\right\rangle\right] \in\left(\mathbb{N}^{|\sigma|}\right)^{\diamond}$, where $\mathfrak{m}^{\nu^{(1)}}, \ldots, \mathfrak{m}^{\nu^{(r)}}$ are the irreducible components of $I_{E}$ with $\operatorname{supp} \nu^{(i)}=\sigma$. Here $I_{E}$ is the monomial ideal of $\mathbb{Q}\left[X_{1}, \ldots, X_{m}\right]$ corresponding to $E$. Combining the various $\varphi(E, \sigma)$ we obtain a quasi-embedding

$$
\mathcal{F}\left(\mathbb{N}^{m}\right) \rightarrow \prod_{\sigma \subseteq\{1, \ldots, m\}}\left(\mathbb{N}^{|\sigma|}\right)^{\diamond}: E \mapsto(\varphi(E, \sigma): \sigma \subseteq\{1, \ldots, m\})
$$

By Proposition 2.8 it follows that

$$
\mathrm{o}\left(\mathcal{F}\left(\mathbb{N}^{m}\right)\right) \leq \bigotimes_{\sigma \subseteq\{1, \ldots, m\}} \mathrm{o}\left(\left(\mathbb{N}^{|\sigma|}\right)^{\diamond}\right)
$$


In order to continue our majorization, we need to bound the type of the Noetherian ordered sets $\left(\mathbb{N}^{|\sigma|}\right)^{\diamond}$. Recall that $\varepsilon_{0}$ is the supremum of the sequence of ordinals $\omega, \omega^{\omega}, \omega^{\omega}, \ldots$; in other words, $\varepsilon_{0}$ is the smallest solution of the equation $\omega^{x}=x$ in ordinals. Given an ordinal $\alpha$ we define

$$
\alpha^{\prime}= \begin{cases}\alpha & \text { if } \alpha<\varepsilon_{0}, \\ \omega \otimes \alpha & \text { if } \alpha \geq \varepsilon_{0} .\end{cases}
$$

We will show:

Lemma 4.1. Let $S$ be a Noetherian ordered set of type $\alpha=\mathrm{o}(S)$. Then $S^{\diamond}$ is Noetherian of type $\mathrm{o}\left(S^{\diamond}\right) \leq \omega^{\alpha^{\prime}}$.

In [39] one finds that $\mathrm{o}\left(S^{*}\right)=\omega^{\omega^{\alpha^{*}}}$ for every Noetherian ordered set $S$ of type $\alpha$. Here

$$
\alpha^{*}= \begin{cases}\alpha-1 & \text { if } 0<\alpha<\omega, \\ \alpha+1 & \text { if } \alpha=\varepsilon+n \text { for some } n<\omega \text { and some } \varepsilon \text { with } \varepsilon=\omega^{\varepsilon}, \\ \alpha & \text { otherwise. }\end{cases}
$$

This yields the cruder upper bound $\mathrm{o}\left(S^{\diamond}\right) \leq \omega^{\omega^{\alpha^{*}}}$. The lemma above was inspired by the following consequence of it, a quantitative version of a wellknown result of B. H. Neumann.

Corollary 4.2 (van den Dries-Ehrlich [43], [44]). Let $\Gamma$ be an ordered abelian group and $S \subseteq \Gamma^{\geq 0}$ well-ordered of order type $\alpha=\mathrm{o}(S)$. Then the monoid $[S]$ generated by $S$ in $\Gamma$ is well-ordered of order type $\leq \omega^{\alpha^{\prime}}$.

Proof. Since $S^{\diamond}$ is the free commutative monoid generated by $S$, we have a natural surjective monoid homomorphism $S^{\diamond} \rightarrow[S]$. This homomorphism is increasing when $S^{\diamond}$ is equipped with the ordering $\leq^{\diamond}$ and $[S]$ with the well-ordering induced from $\Gamma$. The claim now follows from the last lemma and Proposition 2.8(3).

Lemma 4.1 and (4.1) yield the following upper bound on the type of $\mathcal{F}\left(\mathbb{N}^{m}\right)$ :

$$
\mathrm{o}\left(\mathcal{F}\left(\mathbb{N}^{m}\right)\right) \leq \bigotimes_{\sigma \subseteq\{1, \ldots, m\}} \omega^{\omega^{|\sigma|}}=\omega^{\oplus_{\sigma} \omega^{|\sigma|}}=\omega^{(\omega+1)^{\otimes m}},
$$

where $\alpha^{\otimes m}=\alpha \otimes \cdots \otimes \alpha$ ( $m$ times) for $\alpha \in$ On. (If the Cantor normal form of $\alpha$ has leading term $\omega^{\gamma}$, then the leading term of $\alpha^{\otimes m}$ is $\omega^{\gamma m}$. This implies the bound on $\mathrm{o}\left(\mathcal{F}\left(\mathbb{N}^{m}\right)\right)$ given in the introduction.)

Proof of Lemma 4.1. We proceed by transfinite induction on $\alpha$. The case $\alpha=0$ is trivial $(S=\emptyset)$, so let $\alpha>0$. We distinguish two cases. First suppose that $\alpha$ is not additively indecomposable, that is, $\alpha=\alpha_{1} \oplus \alpha_{2}$ for some ordinals $\alpha_{1}, \alpha_{2}<\alpha$. Hence $S$ is a disjoint union $S=S_{1} \cup S_{2}$ with $\mathrm{o}\left(S_{1}\right) \leq \alpha_{1}$ and $\mathrm{o}\left(S_{2}\right) \leq \alpha_{2}$. (Here each $S_{i}$ is equipped with the restriction 
of the ordering of $S$ to $S_{i}$.) We have a bijective increasing map $S_{1}^{\diamond} \times S_{2}^{\diamond} \rightarrow S^{\diamond}$, so

$$
\mathrm{o}\left(S^{\diamond}\right) \leq \mathrm{o}\left(S_{1}^{\diamond} \times S_{2}^{\diamond}\right)=\mathrm{o}\left(S_{1}^{\diamond}\right) \otimes \mathrm{o}\left(S_{2}^{\diamond}\right) \leq \omega^{\alpha_{1}^{\prime}} \otimes \omega^{\alpha_{2}^{\prime}}=\omega^{\alpha^{\prime}}
$$

by Proposition 2.8 and using the induction hypothesis. Now suppose that $\alpha$ is additively indecomposable. It is well-known that then $\alpha$ has the form $\alpha=\omega^{\beta}$ for some $\beta>0$. By Proposition 2.8(1) it suffices to show that $\mathrm{o}\left(\left(S^{\diamond}\right)^{\nsucceq w}\right)<\omega^{\alpha^{\prime}}$ for all $w \in S^{\diamond}$. We show this by induction on $|w|$. For $|w|=0$ there is nothing to show, since then $\left(S^{\diamond}\right)^{\nsupseteq w}=\emptyset$. Suppose $|w|>0$, say $w=\left[s_{0}, \ldots, s_{m-1}\right]$ with $s_{0}, \ldots, s_{m-1} \in S$. We define a map

$$
\psi:\left(S^{\diamond}\right)^{\nsupseteq w} \rightarrow\left(S^{\searrow s_{0}}\right)^{\diamond} \amalg\left(S \times\left(S^{\diamond}\right)^{\searrow w^{\prime}}\right),
$$

where $w^{\prime}=\left[s_{1}, \ldots, s_{m-1}\right]$, as follows: Let $v=\left[t_{0}, \ldots, t_{n-1}\right] \in S^{\diamond}$ with $v \nsupseteq w$. Then either $t_{i} \geq s_{0}$ for all $i$, or $t_{i} \geq s_{0}$ for some $i$, so after reordering the $t$ 's we may assume $t_{0} \geq s_{0}$, and $v^{\prime}=\left[t_{1}, \ldots, t_{n-1}\right] \geq\left[s_{1}, \ldots, s_{n-1}\right]$. In the first case, we put $\psi(v)=v \in\left(S^{\searrow s_{0}}\right)^{\diamond}$, and in the second case, we put $\psi(v)=\left(t_{0}, v^{\prime}\right) \in S \times\left(S^{\diamond}\right)^{\nexists w^{\prime}}$. It is easy to check that $\psi$ is a quasi-embedding. By Proposition 2.8,

$$
\mathrm{o}\left(\left(S^{\diamond}\right)^{\nsucceq w}\right) \leq \mathrm{o}\left(\left(S^{\nsupseteq s_{0}}\right)^{\diamond}\right) \oplus\left(\alpha \otimes \mathrm{o}\left(\left(S^{\diamond}\right)^{\nsupseteq w^{\prime}}\right)\right) .
$$

Put $\gamma=\mathrm{o}\left(S^{\ngtr s_{0}}\right)$, so $\gamma<\alpha$ and hence $\mathrm{o}\left(\left(S^{\ngtr s_{0}}\right)^{\diamond}\right) \leq \omega^{\gamma^{\prime}}<\omega^{\alpha^{\prime}}$ by inductive hypothesis on $\alpha$. By inductive hypothesis on $w$ we have $\delta:=\mathrm{o}\left(\left(S^{\diamond}\right)^{\nsucceq w^{\prime}}\right)$ $<\omega^{\alpha^{\prime}}$. Hence it suffices to show that $\alpha \otimes \delta<\omega^{\alpha^{\prime}}$. Write $\delta$ in Cantor normal form as $\delta=\omega^{\delta_{1}} n_{1}+\cdots+\omega^{\delta_{k}} n_{k}$ with ordinals $\delta_{1}>\cdots>\delta_{k}$ and positive integers $n_{1}, \ldots, n_{k}$. Then the Cantor normal form of $\alpha \otimes \delta$ has leading term $\omega^{\beta \oplus \delta_{1}} n_{1}$. If $\alpha<\varepsilon_{0}$ then $\beta<\omega^{\beta}=\alpha$, and $\delta_{1}<\alpha=\alpha^{\prime}$, hence $\beta \oplus \delta_{1}<\alpha$, since $\alpha$ is additively indecomposable. If $\alpha \geq \varepsilon_{0}$, then $\beta \leq \omega^{\beta}<\omega^{\beta+1}$ and $\delta_{1}<\omega \otimes \alpha=\omega^{\beta+1}$, hence $\beta \oplus \delta_{1}<\omega^{\beta+1}=\omega \otimes \alpha$, since $\omega^{\beta+1}$ is additively indecomposable. In both cases we have $\beta \oplus \delta_{1}<\alpha^{\prime}$, hence $\alpha \otimes \delta<\omega^{\alpha^{\prime}}$ as desired.

REMARK. By Remark 1.11 and Proposition 2.8, the lemma we just proved also implies that $\mathrm{o}\left(S^{\diamond}, \npreceq\right) \leq \omega^{\alpha^{\prime}}$ for any Noetherian ordered set $(S, \leq)$ of type $\alpha$. See [46] for a proof of the slightly better bound $\mathrm{o}\left(S^{\diamond}, \npreceq\right) \leq \omega^{\alpha}$.

Some possibilities for totally ordering monomial ideals. By Lemma 1.6, we have $\mathcal{F}\left(\mathbb{N}^{m}\right) \cong \operatorname{Decr}\left(\mathbb{N}, \mathcal{F}\left(\mathbb{N}^{m-1}\right)\right.$ ) for $m>1$ : Every final segment $F$ of $\mathbb{N}^{m}$ can be written as the disjoint union

$$
F=\left(F_{0} \times\{0\}\right) \cup\left(F_{1} \times\{1\}\right) \cup \cdots \cup\left(F_{j} \times\{j\}\right) \cup \cdots,
$$

where

$$
F_{j}:=\left\{\left(e_{1}, \ldots, e_{m-1}\right) \in \mathbb{N}^{m-1}:\left(e_{1}, \ldots, e_{m-1}, j\right) \in F\right\},
$$


a final segment of $\mathbb{N}^{m-1}$ (possibly empty). In the notation introduced in the proof of Lemma 1.6, $F_{j}=\varphi_{F}(j)$ for all $j \in \mathbb{N}$. We have $\left(F_{0}, F_{1}, \ldots\right) \in$ $\mathcal{F}\left(\mathbb{N}^{m-1}\right)^{(\geq)}$, that is, $F_{0} \subseteq F_{1} \subseteq \cdots$ is an ascending chain of final segments of $\mathbb{N}^{m-1}$ (and hence becomes eventually stationary). Moreover, $F \supseteq G$ if and only if $F_{j} \supseteq G_{j}$ for all $j$, that is, if and only if $\left(F_{0}, F_{1}, \ldots\right) \leq\left(G_{0}, G_{1}, \ldots\right)$ in the ordering of $\mathcal{F}\left(\mathbb{N}^{m-1}\right)^{(\geq)}$. The decomposition (4.3) for final segments of $\mathcal{F}\left(\mathbb{N}^{m}\right)$ can be used to explicitly construct a total ordering $\unlhd$ on $\mathcal{F}\left(\mathbb{N}^{m}\right)$ which extends $\supseteq$. By Corollary 1.8 and Proposition 1.3, this ordering will then be a well-ordering. For the construction, we proceed as follows, by induction on $m$ :

(1) If $m=1$, then $F \unlhd G: \Leftrightarrow F \supseteq G$.

(2) Let $m>1$, and suppose we have already constructed a total ordering $\unlhd$ on $\mathcal{F}\left(\mathbb{N}^{m-1}\right)$. We then put $F \unlhd G$ if and only if

$$
\left(F_{0}, F_{1}, \ldots\right) \unlhd_{\text {lex }}\left(G_{0}, G_{1} \ldots\right)
$$

in the lexicographic ordering on $\mathcal{F}\left(\mathbb{N}^{m-1}\right)^{(\geq)}$induced by $\unlhd$. (That is, $F \unlhd G$ if and only if either $F=G$, or there is $j \in \mathbb{N}$ with $F_{0}=G_{0}, \ldots, F_{j-1}=G_{j-1}, F_{j} \triangleleft G_{j}$.)

By induction on $m$ it follows easily that $F \supseteq G \Rightarrow F \unlhd G$, for all $F, G \in$ $\mathcal{F}\left(\mathbb{N}^{m}\right)$. The empty final segment is the largest and the final segment $\mathbb{N}^{m}$ the smallest element of $\mathcal{F}\left(\mathbb{N}^{m}\right)$.

We shall not try to compute here the order type of $\left(\mathcal{F}\left(\mathbb{N}^{m}\right), \unlhd\right)$ for general $m$. Let us just point out:

LEMMA 4.3. o $\left(\mathcal{F}\left(\mathbb{N}^{2}\right), \unlhd\right)=\omega^{\omega+1}+1$.

In order to see this, suppose that $S$ is a well-ordering. Then the restriction of the lexicographic ordering on $S^{\omega}$ to $S^{(\geq)}$is a well-ordering which extends the product ordering. In the next proposition we compute the order type of $S^{(\geq)}$in terms of the order type of $S$. For $S=\mathcal{F}(\mathbb{N})$ this yields the lemma above. We may assume that $S=\alpha$ is an ordinal.

Proposition 4.4. Let $\alpha$ be an ordinal. Then:

$$
\mathrm{o}\left(\alpha^{(\geq)}\right)= \begin{cases}\alpha & \text { if } \alpha=0 \text { or } \alpha=1, \\ \omega^{\alpha-1}+1 & \text { if } 2 \leq \alpha<\omega \\ \omega^{\alpha} & \text { if } \alpha \geq \omega \text { is a limit } \\ \omega^{\alpha}+1 & \text { if } \alpha \geq \omega \text { is a successor. }\end{cases}
$$

Proof. Clearly o $\left(0^{(\geq)}\right)=0$. Now $\beta^{(\geq)}$is an initial segment of $\gamma^{(\geq)}$for $\beta<\gamma$, so

$$
\mathrm{o}\left(\alpha^{(\geq)}\right)=\bigcup_{\beta<\alpha} \mathrm{o}\left(\beta^{(\geq)}\right) \quad \text { if } \alpha \text { is a limit ordinal. }
$$


Moreover,

$$
(\alpha+1)^{(\geq)}=\bigcup_{i<\omega} B_{i} \cup\{(\alpha, \alpha, \ldots)\},
$$

where $B_{i}$ is the set of decreasing sequences in $\alpha+1$ that begin with exactly $i$ many $\alpha$ 's. Hence each $B_{i}$ is isomorphic to $\alpha^{(\geq)}$(as ordered set), thus

$$
\mathrm{o}\left((\alpha+1)^{(\geq)}\right)=\underbrace{\mathrm{o}\left(\alpha^{(\geq)}\right)+\mathrm{o}\left(\alpha^{(\geq)}\right)+\cdots}_{\omega \text { many times }}+1=\mathrm{o}\left(\alpha^{(\geq)}\right) \omega+1 .
$$

The formula (4.4) follows by transfinite induction, using (4.5) and (4.6).

The well-ordering $\unlhd$ of $\mathcal{F}\left(\mathbb{N}^{m}\right)$ introduced above has several disadvantages. Most severely, from a practical point of view, suppose $F$ and $G$ are two final segments of $\mathbb{N}^{m}$, given in terms of finite sets of generators, and we want to compare $F$ and $G$ with respect to $\unlhd$. So we need to compute representations (4.3) for $F$ and $G$, and lexicographically compare the resulting sequences of final segments of $\mathbb{N}^{m-1}$. This gives rise to a computationally demanding recursion on $m$. Sometimes, however, we have access to the Hilbert-Samuel polynomials of monomial ideals (since they are needed for an auxiliary computation, say). In this case, we may use a variant of the ordering $\unlhd$ for which comparing $F, G \in \mathcal{F}\left(\mathbb{N}^{m}\right)$ can be done in a more efficient way: By Section 2 we obtain a well-ordering $\leq$ of $\mathcal{F}\left(\mathbb{N}^{m}\right)$ extending $\supseteq$ with minimal possible order type $\omega^{m}+1$ by defining

$$
F \leq G: \Leftrightarrow p_{F} \prec p_{G} \text {, or } p_{F}=p_{G} \text { and } F \unlhd G .
$$

This makes it necessary to decide $F \unlhd G$ only to break ties, that is, in case $F$ and $G$ have the same Hilbert-Samuel polynomial.

The Kleene-Brouwer ordering. In the rest of the paper we study another ordering of monomial ideals which has the advantage that comparison of monomial ideals specified by sets of generators is extremely easy.

Definition 4.5. Let $(U, \leq)$ be a totally ordered set. We define the Kleene-Brouwer ordering $\leq_{\mathrm{KB}}$ of the tree $U^{*}$ as follows: If $s=\left(s_{1}, \ldots, s_{m}\right)$, $t=\left(t_{1}, \ldots, t_{n}\right)$, then $s \leq_{\mathrm{KB}} t$ if and only if either

(1) $s \sqsupseteq t$, or

(2) $\left(s_{1}, \ldots, s_{k}\right) \leq_{\text {lex }}\left(t_{1}, \ldots, t_{k}\right)$, where $k=\min \{m, n\}$ and $\leq_{\text {lex }}$ denotes the lexicographic ordering on $U^{k}$.

It it easy to check that $\leq_{\mathrm{KB}}$ is a total ordering on $U^{*}$ extending the initial segment relation $\sqsupseteq$. We refer, e.g., to $[20,(2.12)]$ for a proof of the following fact:

Lemma 4.6. Let $(U, \leq)$ be a well-ordered set and $T$ a tree on $U$. Then $T$ is well-founded if and only if the Kleene-Brouwer ordering restricted to 
$T$ is a well-ordering. (In this case, we write $\mathrm{O}_{\mathrm{KB}}(T)$ for the order type of $\leq_{\mathrm{KB}}$.)

Let now $(S, \leq)$ be a Noetherian ordered set, and fix a total ordering $\leq^{\prime}$ on $S$ extending $\leq$. As in Section 1 we let $\operatorname{Ant}_{\leq^{\prime}}(S)$ be the well-founded tree $\operatorname{Ant}_{\leq^{\prime}}(S):=\left\{\left(s_{1}, \ldots, s_{n}\right) \in S^{*}: s_{i} \| s_{j}\right.$ and $s_{i}<^{\prime} s_{j}$ for all $\left.1 \leq i<j \leq n\right\}$

on $S$. We consider $\operatorname{Ant}_{\leq^{\prime}}(S)$ as an ordered set via the restriction of $\leq_{\mathrm{KB}}$. We define a bijection $\varphi: \mathcal{F}(S) \rightarrow \operatorname{Ant}_{\leq^{\prime}}(S)$ by $\varphi(F)=\left(a_{1}, \ldots, a_{n}\right)$, where $a_{1}, \ldots, a_{n}$ are the minimal generators of $f \in \mathcal{F}(S)$, ordered in increasing order with respect to $\leq$.

Lemma 4.7. The map $\varphi: \mathcal{F}(S) \rightarrow \operatorname{Ant}_{\leq^{\prime}}(S)$ is strictly increasing.

Proof. Let $F \supset G$ be final segments of $S$, and let $a_{1}, \ldots, a_{n}$ and $b_{1}, \ldots, b_{m}$ be the minimal generators of $F$ and $G$, respectively, with $a_{1}<{ }^{\prime} \cdots<a_{n}$ and $b_{1}<^{\prime} \cdots<^{\prime} b_{m}$. We need to show $\left(a_{1}, \ldots, a_{n}\right)<_{\mathrm{KB}}\left(b_{1}, \ldots, b_{m}\right)$. If $\left(b_{1}, \ldots, b_{m}\right) \sqsubset\left(a_{1}, \ldots, a_{n}\right)$ we are done. Otherwise, there exists $r \leq$ $\min \{m, n\}$ such that $a_{1}=b_{1}, \ldots, a_{r-1}=b_{r-1}$ and $a_{r} \neq b_{r}$. Since $F \supset G$, we have $a_{i} \leq b_{r}$ for some $i$. Since $a_{j}=b_{j}$ for $j<r$ and $\left\{b_{1}, \ldots, b_{r}\right\}$ is an antichain, we have $i \geq r$, hence $a_{r} \leq^{\prime} a_{i} \leq b_{r}$. Since $a_{r} \neq b_{r}$ we have $a_{r}<^{\prime} b_{r}$, and therefore $\left(a_{1}, \ldots, a_{n}\right)<_{\mathrm{KB}}\left(b_{1}, \ldots, b_{m}\right)$ as required.

By means of the last lemma, we obtain a well-ordering on $\mathcal{F}\left(\mathbb{N}^{m}\right)$ extending $\supseteq$ as follows: Fix a term ordering $\leq^{\prime}$ on $\mathbb{N}^{m}$. Given final segments $F$ and $G$ of $\mathbb{N}^{m}$, with minimal generators $a_{1}<^{\prime} \cdots<^{\prime} a_{r}$ and $b_{1}<^{\prime} \cdots<^{\prime} b_{s}$ (where $r, s \in \mathbb{N}$ ), define

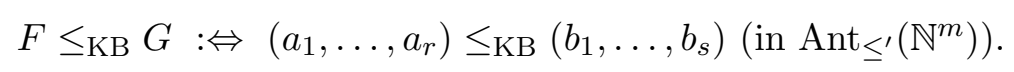

We shall call the well-ordering $\leq_{\mathrm{KB}}$ of $\mathcal{F}\left(\mathbb{N}^{m}\right)$ the Kleene-Brouwer ordering of $\mathcal{F}\left(\mathbb{N}^{m}\right)$ (induced by $\leq^{\prime}$ ), and we put $\mathrm{O}_{\mathrm{KB}}\left(\mathcal{F}\left(\mathbb{N}^{m}\right)\right):=\mathrm{o}_{\mathrm{KB}}\left(\mathrm{Ant}_{<^{\prime}}\left(\mathbb{N}^{m}\right)\right)$. If $I$ and $J$ are monomial ideals in $K\left[X_{1}, \ldots, X_{m}\right]$ (where $K$ is a field) corresponding to final segments $F$ and $G$ of $\mathbb{N}^{m}$, respectively, we put $I \leq_{\mathrm{KB}} J$ if $F \leq_{\text {KB }} G$. This yields a well-ordering on the set of monomial ideals of $K[X]$ which extends $\supseteq$.

For lex-segments, the Kleene-Brouwer ordering induced by the degreelexicographic ordering has an alternative description:

EXAMPLE 4.8. Suppose that $\leq$ is the degree-lexicographic ordering of $\mathbb{N}^{m}$, and let $E=\left(a_{1}, \ldots, a_{r}\right)$ with $a_{1}<^{\prime} \cdots<^{\prime} a_{r}$ be a lex-segment of $\mathbb{N}^{m}$, and let $F=\left(b_{1}, \ldots, b_{s}\right)$ with $b_{1}<^{\prime} \cdots<^{\prime} b_{s}$ be any final segment of $\mathbb{N}^{m}$. Then

$$
E<_{\mathrm{KB}} F \Rightarrow E_{0}=F_{0}, \ldots, E_{d-1}=F_{d-1}, E_{d} \supset F_{d} \text { for some } d \in \mathbb{N} .
$$

Proof. Suppose that $E<_{\mathrm{KB}} F$. If $E \supset F$, we are done. Otherwise, there is $t<\min \{r, s\}$ such that $a_{1}=b_{1}, \ldots, a_{t}=b_{t}, a_{t+1}<^{\prime} b_{t+1}$. Put $d=\left|a_{t+1}\right|$. 
Then $a_{t+1} \notin F_{d}$ : otherwise $a_{t+1} \geq b_{i}$ for some $i$. Since the $a_{1}, \ldots, a_{s}$ form an antichain (with respect to $\leq$ ) and $a_{i}=b_{i}$ for $1 \leq i \leq t$, we have $i>t$ and so $a_{t+1} \geq^{\prime} b_{t+1}$, a contradiction. Moreover $F_{j} \subseteq E_{j}$ for all $0 \leq j \leq d$ : Let $x \in F_{j}$, so $x \geq b_{i}$ for some $i \in\{1, \ldots, s\}$. If $1 \leq i \leq t$ we are done (since $\left.a_{i}=b_{i}\right)$, so suppose $i>t$. Then $x \geq^{\prime} b_{t+1}>^{\prime} a_{t+1}$, so $|x| \geq a_{t+1}=d$, hence $j=d$. Since $E$ is a lex-segment and $x>^{\prime} a_{t+1}$ we get $x \in E_{d}$ as claimed. Finally, we have $E_{j} \subseteq F_{j}$ for $0 \leq j<d$ : If $y \in E_{j}$ for $j \in\{1, \ldots, d-1\}$, then $y \geq a_{i}$ for some $i \in\{1, \ldots, t\}$, hence $y \in F_{j}$.

Historical remark. The Kleene-Brouwer ordering of a tree plays an important role in descriptive set theory and recursion theory. It appears for the first time in the work of Brouwer [9] (in his proof that intuitionistically, every real function is uniformly continuous on closed intervals) and Lusin-Sierpiński [26], and was later used by Kleene [21]. (See the remarks in $[31$, p. 270].) A variant of the Kleene-Brouwer ordering was independently discovered by Ritt in his seminal work on differential algebra, in his definition of the rank of characteristic sets. (See [22, p. 81].)

An upper bound for $\mathrm{OKB}_{\mathrm{KB}}$. We want to investigate the order-theoretic complexity of $\mathrm{O}_{\mathrm{KB}}$. We first establish an upper bound on $\mathrm{O}_{\mathrm{KB}}$.

Notation. Given an ordinal $\alpha$ and a sequence $\left(\alpha_{n}\right)_{n \in \mathbb{N}}$ of ordinals, we write

$$
\alpha=\limsup _{n} \alpha_{n}
$$

if $\alpha=\sup \left\{\alpha_{n}: n \in \mathbb{N}\right\}$, and for every $n_{0}$ and $\beta<\alpha$ there exists $n \geq n_{0}$ with $\beta<\alpha_{n}$. (Equivalently, $\alpha=\lim \sup _{n} \alpha_{n}$ if and only if $\alpha=\sup \left\{\alpha_{n_{i}}: i \in \mathbb{N}\right\}$ for some increasing subsequence $\alpha_{i_{0}} \leq \alpha_{i_{1}} \leq \cdots$ of $\left(\alpha_{n}\right)$.)

In the following, $U$ and $V$ will denote countable sets. For the purpose of this section, let us call a tree $T$ on $U$ universal if $T$ is well-founded, and every node $a$ of $T$ which is not a leaf has infinitely many successors $a_{0}, a_{1}, \ldots$, and $\operatorname{ht}(a)=\lim \sup _{n} \operatorname{ht}\left(a_{n}\right)+1$. Note that the property of being universal is preserved under passing to subtrees.

By Lemma 2.5, if $S$ and $T$ are well-founded trees with $\operatorname{rk}(S) \leq \operatorname{rk}(T)$, then there exists an increasing length-preserving map $S \rightarrow T$. If $T$ is universal, we have the following result (justifying our choice of terminology):

Lemma 4.9. Let $S$ and $T$ be trees on $U$ and $V$, respectively. If $T$ is universal, then the following are equivalent:

(1) $S$ is well-founded with $\operatorname{rk}(S) \leq \operatorname{rk}(T)$.

(2) There exists a length-preserving embedding $S \rightarrow T$.

(3) There exists a strictly increasing map $S \rightarrow T$. 
Proof. We prove $(1) \Rightarrow(2)$ by induction on the rank of $T$, the case $\operatorname{rk}(T)=0$ being trivial. Suppose that $\operatorname{rk}(T)=\alpha+1$ is a successor. Since $T$ is universal, there exists a sequence $\left(b_{n}\right)_{n \in \mathbb{N}}$ of pairwise distinct elements of $V$ such that $\left(b_{0}\right),\left(b_{1}\right), \ldots$ are successors of $\varepsilon$ in $T$ of height $\alpha$. Suppose that $S$ is wellfounded with $\operatorname{rk}(S) \leq \operatorname{rk}(T)$, and let $\left(\left(a_{n}\right)\right)_{n<\lambda}$ (where $\lambda \leq \omega$ and $a_{i} \neq a_{j}$ for all $0 \leq i<j<\lambda$ ) be the successors of the root $\varepsilon$ in $S$. For every $n$, the subtree $S_{\left(a_{n}\right)}$ has rank $\leq \alpha$ and hence can be embedded into $T_{\left(b_{n}\right)}$ by a length-preserving embedding, by induction hypothesis. Hence there exists a length-preserving embedding $a_{n} T_{\left(a_{n}\right)} \rightarrow b_{n} S_{\left(b_{n}\right)}$. Extending the union of these embeddings to a map $S \rightarrow T$ by mapping the root of $S$ to the one of $T$ gives a length-preserving embedding of $S$ into $T$. Finally, suppose that $\operatorname{rk}(T)$ is a limit ordinal. Let $\left(b_{n}\right)_{n \in \mathbb{N}}$ be a sequence of elements of $V$ such that $\sup _{n} \operatorname{rk}\left(T_{\left(b_{n}\right)}\right)=\operatorname{rk}(T)$. Suppose that $S$ is well-founded with $\operatorname{rk}(S) \leq \operatorname{rk}(T)$, and as before let $\left(\left(a_{n}\right)\right)_{n<\lambda}$ (where $\lambda \leq \omega$ and $a_{i} \neq a_{j}$ for all $0 \leq i<j \leq \lambda$ ) be the successors of the root $\varepsilon$ in $S$. For each $0 \leq i<\lambda$ there exists $n_{i} \in \mathbb{N}$ such that $\operatorname{rk}\left(S_{\left(a_{i}\right)}\right) \leq \operatorname{rk}\left(T_{\left(b_{n_{i}}\right)}\right)<\operatorname{rk}(T)$. Using the induction hypothesis we find a length-preserving embedding $a_{i} T_{\left(a_{i}\right)} \rightarrow b_{n_{i}} S_{\left(b_{n_{i}}\right)}$. Again it is not difficult to combine these to obtain a length-preserving embedding $S \rightarrow T$ as required. The implications $(2) \Rightarrow(3)$ and $(3) \Rightarrow(1)$ are clear.

Corollary 4.10. Suppose $U$ is infinite and $T \neq\{\varepsilon\}$ a well-founded tree on $U$. There exists a universal tree $T^{\prime}$ on $U$ with $T \subseteq T^{\prime}$ of the same rank as the tree $T$.

Proof. By Lemma 4.9, it is enough to construct some universal tree on $U$ of $\operatorname{rank} \operatorname{rk}(T)$. This is easy to accomplish by induction on $\operatorname{rk}(T)$.

The order type of $\leq_{\mathrm{KB}}$ is easy to compute for universal trees:

Lemma 4.11. Let $T \neq\{\varepsilon\}$ be a universal tree on a well-ordered set $U$ of order type $\omega$. Then $\mathrm{OKB}(T)=\omega^{\mathrm{rk}(T)}+1$.

Proof. Let $a_{0}<a_{1}<\cdots$ be the successors of the root $\varepsilon$ of $T$, listed according to their order in $U$. Note that

$$
\mathrm{o}_{\mathrm{KB}}(T)=\mathrm{o}_{\mathrm{KB}}\left(T_{0}\right)+\mathrm{o}_{\mathrm{KB}}\left(T_{1}\right)+\cdots+1,
$$

where $T_{n}:=T_{a_{n}}$ is the subtree of $T$ with root at $a_{n}$. We prove the lemma by induction on $\operatorname{rk}(T)>0$. The result is clear if $\operatorname{rk}(T)=1$. Suppose that $\operatorname{rk}(T)=\alpha+1$, where $\alpha>0$. Each $T_{n}$ is universal of rank $\leq \alpha$, so by induction hypothesis, if $T_{n} \neq\{\varepsilon\}$, then $\mathrm{OKB}_{\mathrm{KB}}\left(T_{n}\right)=\omega^{\mathrm{rk}\left(T_{n}\right)}+1$ for all $n$. Because $T$ is universal, there are infinitely many $n \in \mathbb{N}$ such that $\operatorname{rk}\left(T_{n}\right)=\alpha$. Since $\omega^{\beta}+\omega^{\gamma}=\omega^{\gamma}$ whenever $\gamma>\beta$, it follows readily from (4.7) that $\mathrm{OKB}(T)=\omega^{\mathrm{rk}(T)}+1$. Suppose now that $\operatorname{rk}(T)$ is a limit ordinal. Hence $\operatorname{rk}(T)=\sup \left\{\operatorname{rk}\left(T_{n}\right)+1: n \in \mathbb{N}\right\}$ is the limit of a strictly increasing subse- 
quence of $\left(\operatorname{rk}\left(T_{n}\right)\right)$. So by (4.7) and induction hypothesis we have

$$
\mathrm{O} \mathrm{KB}(T)=\left(\bigcup_{n} \omega^{\operatorname{rk}\left(T_{n}\right)}\right)+1=\omega^{\bigcup_{n} \operatorname{rk}\left(T_{n}\right)}+1=\omega^{\operatorname{rk}(T)}+1,
$$

as desired.

Corollary 4.12. For every well-founded tree $T \neq\{\varepsilon\}$ on a well-ordered set $U$ of order type $\omega$, we have $\mathrm{O}_{\mathrm{KB}}(T) \leq \omega^{\mathrm{rk}(T)}+1$.

Clearly it may happen that $\mathrm{OKB}_{\mathrm{KB}}(T)<\omega^{\mathrm{rk}(T)}+1$, for example if $T \neq\{\varepsilon\}$ is finite. Another (infinite) example is given by the tree $T=\{(i, i, \ldots, i)$ $\left.\in \mathbb{N}^{i}: i \in \mathbb{N}\right\}$ of $\operatorname{rank} \omega$ on $\mathbb{N}$.

The order type of the Kleene-Brouwer ordering. We now investigate the order type of the Kleene-Brouwer ordering on $\mathcal{F}\left(\mathbb{N}^{m}\right)$ in the case where $\leq^{\prime}$ has order type $\omega$. By Lemma 2.19 and Corollary 4.12 we obtain the upper bound $\mathrm{O}_{\mathrm{KB}}\left(\mathcal{F}\left(\mathbb{N}^{m}\right)\right) \leq \omega^{\omega^{m-1}}+1$. We will show:

Proposition 4.13. The tree Ant $\leq_{\leq^{\prime}}\left(\mathbb{N}^{m}\right)$ contains a universal tree on $\mathbb{N}^{m}$ with the same rank.

By Lemma 4.11, this immediately yields:

Corollary 4.14. OKB $\left(\mathcal{F}\left(\mathbb{N}^{m}\right)\right)=\omega^{\omega^{m-1}}+1$.

Before we begin the proof, let us introduce some notations: Given an element $\nu \in \mathbb{N}^{m}$ we will denote by $\tau_{\nu}$ the translation

$$
x \mapsto x+\nu: \mathbb{N}^{m} \rightarrow \mathbb{N}^{m},
$$

and given a natural number $n$ we denote by $\iota_{n}$ the map

$$
\nu \mapsto \nu n: \mathbb{N}^{m} \rightarrow \mathbb{N}^{m+1} .
$$

By componentwise application, the map $\tau_{\nu}$ gives rise to a map $\left(\mathbb{N}^{m}\right)^{*} \rightarrow$ $\left(\mathbb{N}^{m}\right)^{*}$ and $\iota_{n}$ gives rise to a map $\left(\mathbb{N}^{m}\right)^{*} \rightarrow\left(\mathbb{N}^{m+1}\right)^{*}$, denoted by the same symbols. We have

$$
\tau_{\nu}\left(\operatorname{Ant}_{\leq^{\prime}}\left(\mathbb{N}^{m}\right)\right) \subseteq \operatorname{Ant}_{\leq^{\prime}}\left(\mathbb{N}^{m}\right) \quad \text { and } \quad \iota_{n}\left(\operatorname{Ant}_{\leq^{\prime}}\left(\mathbb{N}^{m}\right)\right) \subseteq \operatorname{Ant}_{\leq^{\prime}}\left(\mathbb{N}^{m+1}\right) .
$$

For a sequence $a=\left(a_{1}, \ldots, a_{n}\right) \in\left(\mathbb{N}^{m}\right)^{*}$ we put $|a|=\left|a_{1}\right|+\cdots+\left|a_{n}\right|$.

Proof of Proposition 4.13. We proceed by induction on $m=1,2, \ldots$. The case $m=1$ is trivial, since $A_{\leq^{\prime}}(\mathbb{N})$ itself is universal. Suppose that $m>1$ and let $U \subseteq$ Ant $_{\leq^{\prime}}\left(\mathbb{N}^{m-1}\right)$ be a universal tree of rank $\omega^{m-2}$. Put $T:=\operatorname{Ant}_{\leq}\left(\mathbb{N}^{m}\right)$. For any $k \geq 1$, we have

$$
T_{(0, \ldots, 0, k)}=\operatorname{Ant}_{\leq^{\prime}}\left(\mathbb{N}^{m-1} \times\{0, \ldots, k-1\}\right),
$$

hence $\operatorname{rk}\left(T_{(0, \ldots, 0, k)}\right)=\omega^{m-2} k$ by Lemma 2.19. Therefore it suffices to show that $T_{(0, \ldots, 0, k)}$ contains a universal tree of rank $\omega^{m-2} k$. Starting with $V_{0}=\{\varepsilon\}$, 
we construct this tree in $k$ steps. Suppose that $V_{i}(0 \leq i<k)$ is a universal tree which is contained in

$$
T_{(0, \ldots, 0, k)} \cap\left(\mathbb{N}^{m-1} \times\{k-i, \ldots, k-1\}\right)^{*}
$$

and has rank $\omega^{m-2} i$. For each leaf $a$ of $V_{i}$ choose an element $v_{a}$ of $\mathbb{N}^{m-1}$ with $\left|v_{a}\right|>|a|+i+1-k$. It is easy to check that $\left|v_{a}\right|$ is large enough to guarantee that every node of the universal tree $a \iota_{k-i-1}\left(\tau_{v_{a}}(U)\right)$ is an antichain in $\mathbb{N}^{m} \times\{k-i-1, \ldots, k-1\}$ arranged in $\leq^{\prime}$-increasing order. Hence the tree

$$
V_{i+1}:=V_{i} \cup \bigcup_{a} a \iota_{k-i-1}\left(\tau_{v_{a}}(U)\right)
$$

(where the union runs over all leafs $a$ of $V_{i}$ ) is contained in

$$
T_{(0, \ldots, 0, k)} \cap\left(\mathbb{N}^{m-1} \times\{k-i-1, \ldots, k-1\}\right)^{*} .
$$

So $V_{i+1}$ is simply the tree obtained by "implanting" a copy of $U$ (that is, the tree $\left.a \iota_{k-i-1}\left(\tau_{v_{a}}(U)\right)\right)$ at $a$, for each leaf $a$ of $V_{i}$. It is immediate that each non-leaf node of $V_{i+1}$ has $\omega$ many successors. Note also that the heights of nodes of $V_{i+1}$ that are coming from $V_{i}$ will increase by $\operatorname{rk}(U)$ while the heights of nodes coming from $U$ will remain unchanged: $\mathrm{ht}_{V_{i+1}}(v)=\mathrm{ht}_{V_{i}}(v)+\operatorname{rk}(U)$ if $v \in V_{i}$ and $\operatorname{ht}_{V_{i+1}}(v)=\operatorname{ht}_{U}(u)$ for $v=a \iota_{k-i-1}\left(\tau_{v_{a}}(u)\right)$, where $a$ is a leaf of $V_{i}$ and $u \in U$. This observation clearly implies the limsup condition for $V_{i+1}$. Hence $V_{i+1}$ is universal and

$$
\operatorname{rk}\left(V_{i+1}\right)=\operatorname{rk}\left(V_{i}\right)+\operatorname{rk}(U)=\omega^{m-2}(i+1) .
$$

The last tree $V_{k}$ constructed in this way has the desired properties.

Combining Corollary 4.14 and (4.2) we obtain as promised our estimates on the type of the ordered set of monomial ideals:

Corollary 4.15. $\omega^{\omega^{m-1}}+1 \leq \mathrm{o}\left(\mathcal{F}\left(\mathbb{N}^{m}\right)\right) \leq \omega^{(\omega+1)^{\otimes m}}$

\section{References}

[1] U. Abraham, A note on Dilworth's theorem in the infinite case, Order 4 (1987), $107-125$.

[2] U. Abraham and R. Bonnet, Hausdorff's theorem for posets that satisfy the finite antichain property, Fund. Math. 159 (1999), 51-69.

[3] M. Aschenbrenner and R. Hemmecke, A finiteness theorem in stochastic integer programming, in preparation, 2003.

[4] M. Aschenbrenner and W. Y. Pong, The differential order, in preparation, 2003.

[5] H. Bachmann, Transfinite Zahlen, Ergeb. Math. Grenzgeb. 1, Springer, Berlin, 1955.

[6] T. Becker and V. Weispfenning, Gröbner Bases, Grad. Texts in Math. 141, Springer, New York, 1993.

[7] R. Bonnet et M. Pouzet, Extension et stratification d'ensembles dispersés, C. R. Acad. Sci. Paris Sér. A-B 268 (1969), A1512-A1515. 
[8] G. Brookfield, The length of Noetherian modules, Comm. Algebra 30 (2002), 31773204.

[9] L. E. J. Brouwer, Beweis, daß jede volle Funktion gleichmässig stetig ist, Nederl. Akad. Wetensch. Proc. Ser. B 27 (1924), 189-193.

[10] W. Bruns and J. Herzog, Cohen-Macaulay Rings, Cambridge Stud. Adv. Math. 39, Cambridge Univ. Press, Cambridge, 1993.

[11] D. de Jongh and R. Parikh, Well-partial orderings and hierarchies, Indag. Math. 39 (1977), 195-207.

[12] N. Dershowitz, Termination of rewriting, J. Symbolic Comput. 3 (1987), 69-115.

[13] R. P. Dilworth, A decomposition theorem for partially ordered sets, Ann. of Math. (2) 51 (1950), 161-166.

[14] T. Dubé, The structure of polynomial ideals and Gröbner bases, SIAM J. Comput. 19 (1990), 750-775.

[15] D. Eisenbud, Commutative Algebra with a View Toward Algebraic Geometry, Grad. Texts in Math. 150, Springer, New York, 1995.

[16] R. Fraïssé, Theory of Relations, revised ed., Stud. Logic Found. Math. 145, NorthHolland, Amsterdam, 2000.

[17] H. Friedman, The Ackermann function in elementary algebraic geometry, manuscript, 1999.

[18] J. H. Gallier, What's so special about Kruskal's theorem and the ordinal $\Gamma_{0}$ ? A survey of some results in proof theory, Ann. Pure Appl. Logic 53 (1991), 199-260.

[19] G. Higman, Ordering by divisibility in abstract algebras, Proc. London Math. Soc. (3) 2 (1952), 326-336.

[20] A. S. Kechris, Classical Descriptive Set Theory, Grad. Texts in Math. 156, Springer, New York, 1995.

[21] S. C. Kleene, On the forms of the predicates in the theory of constructive ordinals. II, Amer. J. Math. 77 (1955), 405-428.

[22] E. Kolchin, Differential Algebra and Algebraic Groups, Pure and Appl. Math. 54, Academic Press, New York-London, 1973.

[23] M. V. Kondratieva, A. B. Levin, A. V. Mikhalev, and E. V. Pankratiev, Differential and Difference Dimension Polynomials, Math. Appl. 461, Kluwer, Dordrecht, 1999.

[24] I. Kříž and R. Thomas, Ordinal types in Ramsey theory and well-partial-ordering theory, in [34], 57-95.

[25] J. B. Kruskal, The theory of well-quasi-ordering: A frequently discovered concept, J. Combin. Theory Ser. A 13 (1972), 297-305.

[26] N. Lusin et W. Sierpiński, Sur un ensemble non mesurable B, J. Math. Pures Appl. (9) 2 (1923), 53-72.

[27] F. S. Macaulay, Some properties of enumeration in the theory of modular systems, Proc. London Math. Soc. 26 (1927), 531-555.

[28] D. Maclagan, Antichains of monomial ideals are finite, Proc. Amer. Math. Soc. 129 (2001), 1609-1615.

[29] U. Martin and E. Scott, The order types of termination orderings on monadic terms, strings and multisets, J. Symbolic Logic 62 (1997), 624-635.

[30] G. Moreno Socías, Length of polynomial ascending chains and primitive recursiveness, Math. Scand. 71 (1992), 181-205.

[31] Y. N. Moschovakis, Descriptive Set Theory, Stud. Logic Found. Math. 100, NorthHolland, Amsterdam, 1980.

[32] C. St. J. A. Nash-Williams, On well-quasi-ordering finite trees, Proc. Cambridge Philos. Soc. 59 (1963), 833-835.

[33] —, On well-quasi-ordering infinite trees, ibid. 61 (1965), 697-720. 
[34] R. Nešetřil and V. Rödl (eds.), Mathematics of Ramsey Theory, Algorithms Combin. 5, Springer, Berlin, 1990.

[35] M. Perles, On Dilworth's theorem in the infinite case, Israel J. Math. 1 (1963), 108-109.

[36] R. Rado, Partial well-ordering of sets of vectors, Mathematika 1 (1954), 89-95.

[37] L. Robbiano, Term orderings on the polynomial ring, in: EUROCAL '85, Vol. 2 (Linz, 1985), Lecture Notes in Comput. Sci. 204, Springer, Berlin, 1985, 513-517.

[38] C. J. Rust, Rankings of Derivatives for Elimination Algorithms and Formal Solvability of Analytic Partial Differential Equations, Ph.D. thesis, Univ. of Chicago, 1998.

[39] D. Schmidt, Well-Partial Orderings and Their Maximal Order Types, Habilitationsschrift, Math. Inst., Univ. Heidelberg, 1979.

[40] S. Simpson, Subsystems of Second Order Arithmetic, Perspectives in Math. Logic, Springer, Berlin, 1999.

[41] W. Sit, Well ordering of certain numerical polynomials, Trans. Amer. Math. Soc. 212 (1975), 37-45.

[42] B. Sturmfels, Gröbner Bases and Convex Polytopes, Univ. Lecture Ser. 8, Amer. Math. Soc., Providence, RI, 1996.

[43] L. van den Dries and P. Ehrlich, Fields of surreal numbers and exponentiation, Fund. Math. 167 (2001), 173-188.

[44] —, 一, Erratum to [43], ibid. 168 (2001), 295-297.

[45] J. van der Hoeven, Asymptotique automatique, Ph.D. thesis, École Polytechnique, Paris, 1997.

[46] A. Weiermann, Proving termination for term rewriting systems, in: Computer Science Logic (Berne, 1991), Lecture Notes in Comput. Sci. 626, Springer, Berlin, 1992, 419-428.

[47] E. S. Wolk, Partially well ordered sets and partial ordinals, Fund. Math. 60 (1967), $175-186$.

Department of Mathematics

University of California at Berkeley

Evans Hall

Berkeley, CA 94720, U.S.A.

E-mail: maschenb@math.berkeley.edu
Department of Mathematics California State University Dominguez-Hills 1000 E. Victoria Street

Carson, CA 90747, U.S.A. E-mail: pong@math.csudh.edu

Received 10 April 2003;

in revised form 22 February 2004 